\title{
Probiotics, Prebiotics, Synbiotics and Dental Caries. New Perspectives, Suggestions, and Patient Coaching Approach for a Cavity-Free Mouth
}

\author{
Markos Amargianitakis ${ }^{1}$ (D), Maria Antoniadou ${ }^{1}$ (D) Christos Rahiotis ${ }^{1}$ (D) and Theodoros $\operatorname{Varzakas}^{2, *(D)}$ \\ 1 Dental School, National and Kapodistrian University of Athens, 11527 Athens, Greece; \\ markosamargian@gmail.com (M.A.); mantonia@dent.uoa.gr (M.A.); craxioti@dent.uoa.gr (C.R.) \\ 2 Department of Food Science and Technology, University of Peloponnese, 24100 Kalamata, Greece \\ * Correspondence: t.varzakas@uop.gr
}

check for updates

Citation: Amargianitakis, M.; Antoniadou, M.; Rahiotis, C.; Varzakas, T. Probiotics, Prebiotics, Synbiotics and Dental Caries. New Perspectives, Suggestions, and Patient Coaching Approach for a Cavity-Free Mouth. Appl. Sci. 2021, 11, 5472. https://doi.org/10.3390/ app11125472

Academic Editor: Mary Anne Melo

Received: 26 April 2021

Accepted: 9 June 2021

Published: 12 June 2021

Publisher's Note: MDPI stays neutral with regard to jurisdictional claims in published maps and institutional affiliations.

Copyright: (c) 2021 by the authors. Licensee MDPI, Basel, Switzerland. This article is an open access article distributed under the terms and conditions of the Creative Commons Attribution (CC BY) license (https:/ / creativecommons.org/licenses/by/ $4.0 /)$.

\begin{abstract}
Probiotic therapy forms a new strategy for dental caries prevention. Probiotic microorganisms possess the ability to displace cariogenic microorganisms and colonize the oral cavity. They can produce various antimicrobial substances such as bacteriocins, bacteriocin-like peptides, lactic acid, and hydrogen peroxide. Dairy products may be ideal for probiotic administration in dental patients. Many other means have been proposed, primarily for those allergic to dairy components, such as capsules, liquid form, tablets, drops, lozenges, sweetened cakes, and ice creams. The last two forms can be used in a coaching approach for children and elderly patients who find it difficult to avoid sugary beverages in their daily routine and benefit from the suggestion of easy, cheap, and common forms of delicacies. In caries prevention, the concept of the effector strain is already considered an integral part of the contemporary caries cure or prevention strategy in adults. Adults, though, seem not to be favored as much as children at early ages by using probiotics primarily due to their oral microbiome's stability. In this non-systematic review we describe the modes of action of probiotics, their use in the cariology field, their clinical potential, and propose options to prevent caries through a patient coaching approach for the daily dental practice.
\end{abstract}

Keywords: probiotics; prebiotics; synbiotics; dental caries; effector strain; prevention; oral health

\section{Introduction}

Dental caries is a multifactorial disease that occurs because of the ecological imbalance between the inorganic components of the hard dental tissues and biofilms [1]. It is the most widespread disease worldwide, with a prevalence approaching $91 \%$ of the adult population [2-5]. This trend is depicted particularly in USA's national expenditures on oral biofilm-associated diseases, which have surpassed the corresponding expenditures for heart conditions since 2006 [3]. In general, the human microbiome is in balancesymbiosis-with its host, the human body. However, the use of antibiotics seems to cause serious adverse effects, such as damage to the desired oral microbiome, pathogen resistance, and oral cavities more prone to dental caries, among other things [6,7]. For this reason, a newly derived and preventively oriented method, probiotic therapy (i.e., the use of desired and harmless microorganisms) has been gaining ground for the past few years. These facts requisite to form new strategies for dental caries prevention, especially in the post-COVID-19 pandemic recession-era worldwide.

In dentistry, probiotics utilization is being focused on advancing oral health by forestalling caries' and periodontal diseases' establishment [8]. In caries management, probiotics' rationale is that probiotic microorganisms possess the ability to displace cariogenic microorganisms and colonize the oral cavity $[9,10]$. In this review study, we focus our interest on the modes of action of probiotics as much as on the scientific effort from the advent of probiotics in cariology, until today. We also highlight some considerations regarding 
their clinical potential in daily use and propose simple options to prevent caries experience or aggravation through a patient coaching approach for the daily practice.

\section{Nomenclature}

Probiotics were discovered in 1907, from the observation of the Nobel laureate in Immunology and Russian bacteriologist Ilya Ilyich Metchnikoff, that certain bacteria promote human intestinal health [11]. Since then, much has changed in probiotic nomenclature and its perspectives [12-16]. Today, probiotics are defined as "live microorganisms, which when administered in adequate amounts, confer a health benefit on the host" by the World Health Organization (WHO) and the United Nations Food and Agriculture Organization (FAO) (2002) [17]. The term 'prebiotics', on the contrary, is used to describe "a selectively fermented ingredient that allows specific changes, both in the composition and/or activity in the resident microflora, that confers benefits upon host wellbeing and health" [18]. The term 'synbiotic' is applied to products containing probiotics and prebiotics (Figure 1) [19].

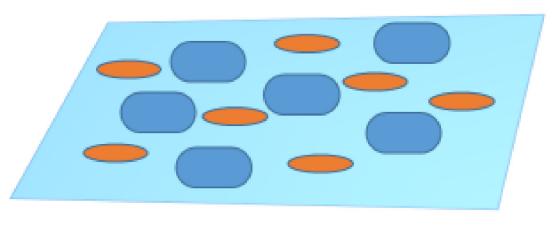

Probiotics and pathogenic microorganisms' coexistence in the oral cavity in the absence of prebiotics

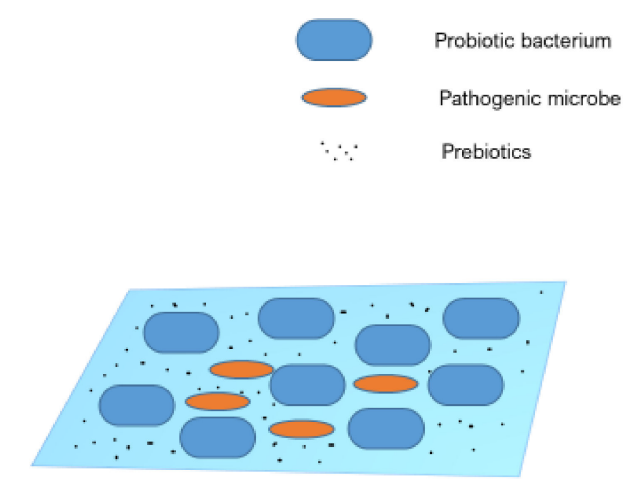

Probiotics and pathogenic microorganisms' coexistence in the oral cavity in the presence of prebiotics

Figure 1. Synbiotics are products containing both probiotics and prebiotics.

It should be noted that fermented foods, although consisting of many microorganisms serving as probiotics, do not follow the cause explained for probiotics [10]. Fermented foods comprise edible products in which microbial activity is necessary to acquire stability, safety, and sensory properties. This is accomplished due to the ability of certain microorganisms (i.e., fermentation microorganisms/microbiomes) to decompose carbohydrates, thereby producing metabolites, such as lactic acid (lactic acid bacteria and Enterobacteriaceae), acetic acid (Acetobacter spp., Gluconobacter spp., Bacillus subtilis and yeasts), ethanol (heterofermentative lactic acid bacteria, Enterobacteriaceae, yeasts etc.), carbon dioxide, hydrogen peroxide, bacteriocins and antimicrobial peptides, which act alone or collectively to inhibit spoilage and the growth of several pathogens. Therefore, the microbiomes used for fermentation do not aim primarily to alter a human's microflora, even though many probiotic strains (e.g., Lactobacilli spp.) utilized in general medicine and dentistry have been derived from the fermentation industry.

\section{Modes of Action of Oral Probiotics}

Several microorganisms serve as oral probiotics. Probiotics that have been used in clinical trials are classified regarding the genus, the species, and the strain (Table 1). 
Table 1. Microorganisms are serving as oral probiotics.

\begin{tabular}{|c|c|c|}
\hline Genus & Species & Strain \\
\hline \multirow[t]{11}{*}{ Lactobacillus } & rhamnosus & GG (ATCC 53103) [20-23], het 70 [24], LB21 [25], LC 705 [22] \\
\hline & reuteri & $\begin{array}{c}\text { ATCC 55,730 (SD2112) [26-28], ATCC PTA } 5289 \text { [29-32], } \\
\text { DSM 17,938 [29-32] }\end{array}$ \\
\hline & casei & Shirota [33] \\
\hline & paracasei & F19 [34], GMNL-33 [35], SD1 [36-38] \\
\hline & achidophilus & ATCC 4356 [39], La-5 [40] \\
\hline & salivarius & TI 2711 [41], WB21 [41] \\
\hline & brevis & CD2 [42] \\
\hline & bifidum [43] & \\
\hline & bulgaricus [43] & \\
\hline & sporogens [43] & \\
\hline & thermophilus [43] & \\
\hline \multirow[t]{3}{*}{ Bifidobacterium } & animalis lactis & BB-12 (ATCC 27536) [40,44-46], DN-173010 [47,48] \\
\hline & bifidum & ATCC 29,521 [39] \\
\hline & longum [49] & \\
\hline \multirow[t]{6}{*}{ Streptococci } & mutans & A2JM [50] \\
\hline & rattus & $\mathrm{JH} 145^{\mathrm{TM}}[51,52]$ \\
\hline & oralis & $\mathrm{KJ}^{\mathrm{TM}}[52]$ \\
\hline & uberis & $\mathrm{KJ} 2^{\mathrm{TM}}[52]$ \\
\hline & dentisani & CECT7746 [53] \\
\hline & salivarius & M18 [54] \\
\hline Bacillus & coagulans [55] & \\
\hline
\end{tabular}

The probiotics' mechanisms of action generally have not been precisely determined [56]. Nevertheless, in general, the three main modes through which probiotics exert their action are (a) modulation of host's defense (b) direct destruction of pathogens, and (c) indirect removal of pathogens.

Probiotics and their extracellular products are found to interact with the host's mucous cells, determining in a strain-specific manner the cytokines' and chemokine's production, which leads to the enhanced phagocytic activity of macrophages, neutrophils, and Natural Killer (NK) cells [57]. For example, B. lactis Bb-12, L. rhamnosus GG, and L. acidophilus La1 increase the phagocytic capacity of leukocytes [58-61]. Probiotics, however, manipulate not only innate immunity but also stimulate adaptive immunity by increasing IgA levels in the serum and regulating the development of $\mathrm{T}$ helper cells and the proportion of Th1/Th2 cells [57,62-64]. In the oral environment, much less has been elucidated [56]. Indeed, specific probiotics inhibit the interleukin-8 (IL-8) response of the oral mucous cells caused by some periodontal pathogens as much as other inflammatory biomarkers, such as prostaglandin E2 (PGE2) [65-67]. Still, no alteration in salivary IgA levels has been observed [68]. Additionally, L. paracasei has been proved to augment the detectable counts of a defensin [69], salivary human neutrophil peptide 1-3 [70].

Probiotic bacteria can produce various antimicrobial substances, such as bacteriocins, bacteriocin-like peptides, lactic acid, and hydrogen peroxide. All of the above have an immediate effect on the host's microbiome, as they preconceive the death of specific pathogens, while the producer strains survive [71-73]. For example, L. rhamnosus GG secretes a broadspectrum antimicrobial substance affecting many Gram-positive (Streptococci, Lactobacilli, Clostridium spp.) and Gram-negative bacteria (E. coli, Bacillus fragilis) [74]. L. reuteri produces 
reuterin and reutericyclin $[75,76]$, which exert their antimicrobial properties by inducing oxidative stress and altering the transmembrane $\Delta \mathrm{pH}$ in target cells $[77,78]$, respectively.

The indirect effects of probiotic microorganisms on the host's microbiome have to do with the phenomenon of competing with the pathogenic bacteria either for an adhesion niche or for essential nutrients [79-82]. Whenever salutary strains preoccupy potential sites of pathogens' adhesion, disease establishment is subverted [56]. The same happens when probiotic organisms excrete certain bio-surfactants that impede pathogenic bacteria's adhesion or when they modify the salivary pellicle per se [82,83]. The previous adhesion sites are altered in a direction rendering them not probable for pathogens to establish.

\section{Caries Pathogenesis}

For a thorough perception of the role of probiotics in caries prevention and therapy, an analysis of the mechanism via which caries lesions develop is imperative. The oral cavity constitutes a habitat for a wide variety of microorganisms [84]. The latter are found to colonize both the oral mucosa and stable surfaces, such as teeth, fixed and removable prosthodontic appliances, etc. It is those microorganisms that colonize tooth surfaces to which dental caries is attributed. These microorganisms conglomerate, thereby constituting a complex, tolerant antimicrobial agent mass called 'oral biofilm' or 'dental plaque'.

Oral biofilms are made up of a plethora of microorganisms. Some of them are harmless when present in the oral cavity, and some others possess a facultative pathogenic potential, which is called 'opportunistic pathogens' [85]. According to the 'Ecological Plaque Hypothesis', in the presence of health, all of them are in a state of symbiosis with each other as much as with the host. In fact, they play a crucial role in the host's health. Caries disease results from this symbiotic relationship's subversion, where a shift toward pathogens occurs, a state characterized as dysbiosis. In that case, sugars consumed through diet are taken up by pathogenic bacteria and are metabolized to lactic acid. Acids produced by dental plaque solubilize apatite crystals of the hard dental tissues (i.e., demineralization). Once acidic residues are removed, remineralization occurs (Figure 2).

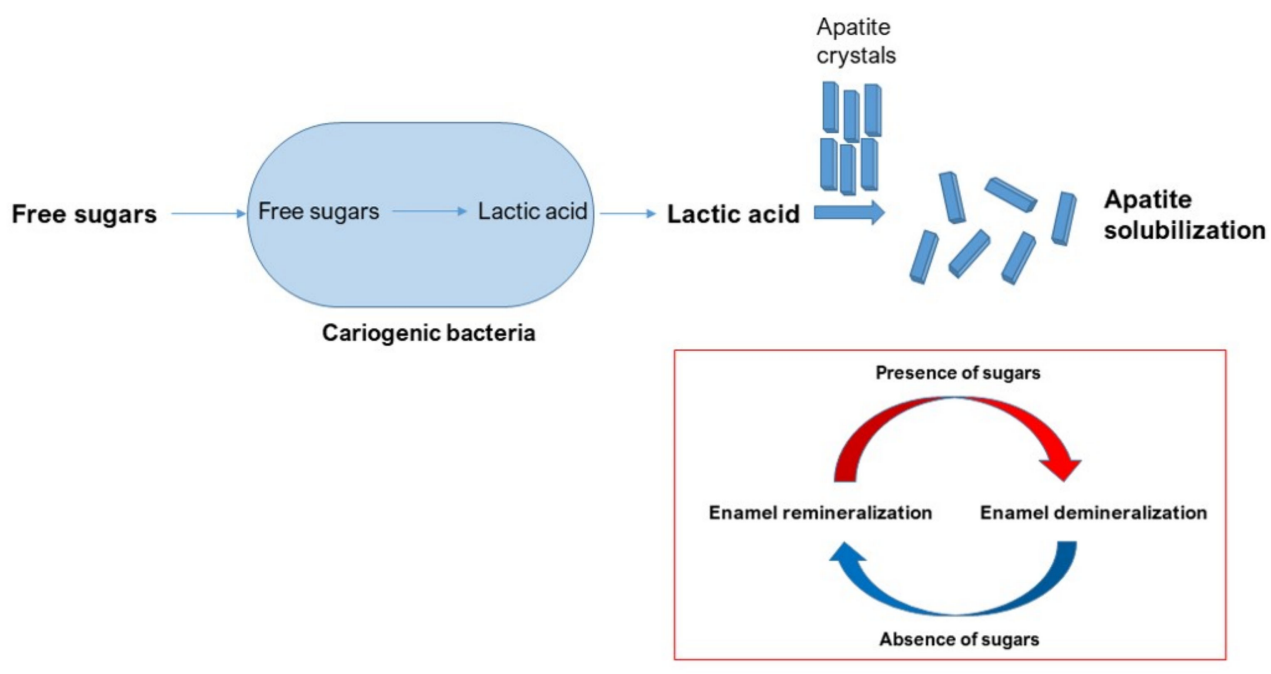

Figure 2. The process of caries development.

However, caries onset is not as simple as presented above. Many factors interplay among the host (salivary flow, apatite solubility), its oral microbiome synthesis, and the type of nutrients being taken up (high/low-sugar diet) [86]. Thus, the effects of acids produced during a sugar-rich diet of low frequency can be neutralized by saliva or alkali produced in the dental plaque, meaning that demineralization and remineralization phenomena are in equilibrium. In the case that a sugar-abundant diet is consumed more frequently, the constant low-pH conditions exert evolutionary pressure toward acid-adapted and acidogenic bacteria, such as Streptococci and Actinomycetes. If this frequency is even higher, 
the most efficient acid-tolerating and acid-producing bacteria dominate. In the last two cases, demineralization of the tooth surfaces prevails at the expense of remineralization [85].

Several microorganisms have been correlated with different types, stages, and sites of cariogenesis [84]. The complex microbial composition of cavities at various stages is not consistent with the specific plaque hypothesis and supports a polymicrobial origin. Nowadays, dental caries cannot be considered a classical infectious disease that follows the conventional Koch's model. The microbial 'players' involved change through time depending on the tissue affected, and multiple species are responsible for the progressing lesion at different stages of the caries process [87].

\section{Caries Management with Probiotics}

Today, probiotics seem to be a salutary, newly derived method to control dental caries $[9,10]$. The rationale of probiotics use in caries management is that probiotic microorganisms can expel cariogenic microorganisms and colonize the oral cavity.

A wide variety of clinical trials have been conducted to examine probiotics' effect on dental caries and oral microflora. These studies are summarized in Table 2.

Among most of these trials, dairy products remained a common denominator as an administration milieu [46-48]. Milk's colloidal nature seems to be enamel protective [88], as it contains organic and inorganic compounds assisting in compensating cariogenic challenges [89]. Another substrate of dairy formulations, calcium lactate, also possesses anti-cariogenic properties [90]. Therefore, dairy products may be ideal for probiotic administration. However, many other means have been proposed to serve as probiotic administration agents, especially for those allergic to dairy components. Among those means stand capsules or liquid form [43], specially prepared straws or tablets [27,35,41], drops [29], lozenges [54], and even sweetened cakes [55] and ice creams [44].

The first-ever study introducing probiotics to dental clinical practice was that of Meurman and his colleagues [20]. They suggested that Lactobacillus Gorbach-Goldin (GG) - LGG, a strain earlier isolated by Gorbach and Goldin [91], can colonize the human oral cavity. Nonetheless, its long-term beneficial effect on oral health and the resulting alterations in oral microflora remained unilluminated. Näse et al. investigated L. rhamnosus GG for its in vivo long-term effect (7-month period) on dental caries [21,92]. They reported no significant changes in $S$. mutans salivary counts and caries prevalence occurred, but a significantly lower caries-risk than in the control group.

Following these results, Ahola et al. investigated the short-term anticaries effect of a cheese containing the same Lactobacillus rhamnosus strain plus L. rhamnosus LC 705 [22]. After a 3-week period, no statistically significant difference in $S$. mutans counts between the intervention and the control group occurred. However, the probiotic-containing cheese was found to exert its enhanced anticaries potential during the post-treatment period, as the $S$. mutans levels in saliva were significantly reduced.

In 2003, Montalto et al. examined two extra facets of probiotics intended for oral administration [43]. Probiotic species tested in this study were L. sporogens $16 \%$, L. bifidum $12 \%$, L. bulgaricus $12 \%$, L. thermophilus $18 \%$, L. acidophilus $20 \%$, L. casei $10 \%$, and L. rhamnosus $12 \%$. The probiotics used, independently of the administration's milieu, were found to increase Lactobacilli salivary counts in contrast to S. mutans. In fact, capsule and liquid forms were found to lead to equivalent results. The only practical difference between these two types of administration is that Lactobacilli strains diffused into the oral cavity in the liquid state. Thus, a systemic effect of oral probiotics was suggested.

In 2004, Nikawa et al. highlighted the selective bactericidal influence of $L$. reuteri SD2112 (ATCC55730) on S. mutans in vitro and in vivo [26]. The individuals consumed a cup (95 g) of placebo yoghurt (S. thermophilus and L. bulgaris) once a day for two weeks during lunchtime in the first group. The following 2 weeks, a probiotic yoghurt containing L. reuteri and S. thermophilus was administrated in the same terms. In the second group, the opposite regimen was followed. Ultimately, both types of treatment significantly reduced the $S$. mutans salivary carriage, compared to its baseline values for each group. 
Table 2. Clinical trials around oral probiotics utilized without aiming to preemptively colonize the oral cavity of subject.

\begin{tabular}{|c|c|c|c|c|c|c|c|c|}
\hline $\begin{array}{l}\text { Baseline } \\
\text { Condition }\end{array}$ & $\begin{array}{l}\text { Type of } \\
\text { Study }\end{array}$ & $\begin{array}{l}\text { Patient } \\
\text { Type }\end{array}$ & $\begin{array}{l}\text { Baseline } \\
\text { Condition }\end{array}$ & Study Groups & Treatment & $\begin{array}{l}\text { Probiotic } \\
\text { Strains }\end{array}$ & $\begin{array}{l}\text { Strain Con- } \\
\text { centration }\end{array}$ & Results \\
\hline $\begin{array}{l}\text { Meurman } \\
\text { et al. } \\
\text { (1994) [20] }\end{array}$ & $\begin{array}{l}\text { Cohort } \\
\text { study }\end{array}$ & $\begin{array}{c}\text { Dental } \\
\text { students } \\
\text { (mean age } \\
25 \text { years) }\end{array}$ & Healthy & 1 test group $(n=$ & $\begin{array}{c}2 \times 250 \mathrm{~g} \text { of probiotic } \\
\text { yoghurt/day }\end{array}$ & $\begin{array}{l}\text { Lactobacillus } \\
\text { rhamnosus GG } \\
\text { ATCC } 53103\end{array}$ & $\begin{array}{c}1 \times 10^{8} \\
\mathrm{CFU} / \mathrm{mL}\end{array}$ & $\begin{array}{l}\text { LGG showed distinct } \\
\text { growth in } 8 \text { of } 9 \text { subjects } \\
2 \text { weeks after treatment } \\
\text { discontinuation. }\end{array}$ \\
\hline $\begin{array}{l}\text { Näse et al. } \\
\text { (2001) [21] }\end{array}$ & $\begin{array}{l}\text { Randomized, } \\
\text { double- } \\
\text { blind, } \\
\text { placebo- } \\
\text { controlled }\end{array}$ & $\begin{array}{c}\text { Daycare } \\
\text { children } \\
\text { (1-6 years } \\
\text { old })\end{array}$ & $\begin{array}{l}\text { Healthy } \\
\text { Caries }\end{array}$ & $\begin{array}{c}\text { Test }(n=231) \\
\text { Control }(n=220)\end{array}$ & $\begin{array}{c}5 \times( \pm 250 \mathrm{~mL}) \text { of } \\
\text { probiotic milk/week } \\
\text { during a } 7-\mathrm{month} \\
\text { period } \\
5 \times( \pm 250 \mathrm{~mL}) \text { of } \\
\text { placebo milk/week } \\
\text { for } 7 \text { months }\end{array}$ & $\begin{array}{l}\text { Lactobacillus } \\
\text { rhamnosus GG } \\
\text { ATCC } 53103\end{array}$ & $\begin{array}{c}5-10 \times 10^{5} \\
\mathrm{CFU} / \mathrm{mL}\end{array}$ & $\begin{array}{l}\text { No significant } \\
\text { differences in caries and } \\
\text { MS scores. Significantly } \\
\text { reduced caries-risk in } \\
\text { the probiotic group, } \\
\text { especially in the 3- to } \\
\text { 4-year-old children. }\end{array}$ \\
\hline $\begin{array}{c}\text { Ahola } \\
\text { et al. } \\
(2002) \text { [22] }\end{array}$ & $\begin{array}{l}\text { Randomized, } \\
\text { double- } \\
\text { blind, } \\
\text { placebo- } \\
\text { controlled }\end{array}$ & $\begin{array}{c}\text { Young } \\
\text { adults } \\
(18-35 \\
\text { years old })\end{array}$ & Healthy & $\begin{array}{c}\text { Test }(n=38) \\
\text { Control }(n=36)\end{array}$ & $\begin{array}{c}5 \times 15 \mathrm{~g} \text { of probiotic } \\
\text { cheese/day for } \\
3 \text { weeks } \\
5 \times 15 \mathrm{~g} \text { of placebo } \\
\text { cheese/day for } \\
3 \text { weeks }\end{array}$ & $\begin{array}{l}\text { Lactobacillus } \\
\text { rhamnosus GG } \\
\text { ATC 53103 } \\
\text { Lactobacillus } \\
\text { rhamnosus LC } \\
705\end{array}$ & $\begin{array}{l}1.9 \times 10^{7} \\
\mathrm{CFU} / \mathrm{g} \\
1.2 \times 10^{7} \\
\mathrm{CFU} / \mathrm{g}\end{array}$ & $\begin{array}{c}\text { No significant } \\
\text { difference in MS and } \\
\text { yeast counts during } \\
\text { intervention. } \\
\text { Significantly reduced } \\
\text { MS scores and a } \\
\text { tendency toward fewer } \\
\text { patients with high } \\
\text { Lactobacilli counts in } \\
\text { the probiotic group } \\
\text { during the } \\
\text { post-treatment period. }\end{array}$ \\
\hline
\end{tabular}

\begin{tabular}{|c|c|c|c|c|c|c|c|c|}
\hline \multirow{7}{*}{$\begin{array}{l}\text { Montalto } \\
\text { et al. } \\
\text { (2004) [43] }\end{array}$} & \multirow{7}{*}{$\begin{array}{l}\text { Randomized, } \\
\text { double- } \\
\text { blind, } \\
\text { placebo- } \\
\text { controlled }\end{array}$} & \multirow{7}{*}{$\begin{array}{c}\text { Young } \\
\text { adults } \\
\text { (23-37 } \\
\text { years old) }\end{array}$} & \multirow{7}{*}{ Healthy } & \multirow{7}{*}{$\begin{array}{l}\text { Group A }(n=14) \\
\text { Group B }(n=16) \\
\text { Group C }(n=5)\end{array}$} & \multirow{7}{*}{$\begin{array}{c}\text { (Capsuled probiotics } \\
+ \text { Liquid } \\
\text { placebo)/day for } \\
45 \text { days } \\
\text { (Liquid probiotics }+ \\
\text { capsuled } \\
\text { placebo)/day for } \\
45 \text { days } \\
\text { (Liquid and capsuled } \\
\text { placebo)/day for } \\
45 \text { days }\end{array}$} & \multirow{7}{*}{$\begin{array}{l}\text { L. sporogens } \\
\text { L. bifidum } \\
\text { L. bulgaricus } \\
\text { L. thermophilus } \\
\text { L. acidophilus } \\
\text { L. casei } \\
\text { L. rhamnosus }\end{array}$} & $16 \%$ & \multirow{7}{*}{$\begin{array}{c}\text { Significant increase in } \\
\text { Lactobacilli groups in } \\
\text { both probiotic groups. } \\
\text { No change in MS counts } \\
\text { in all groups. }\end{array}$} \\
\hline & & & & & & & $12 \%$ & \\
\hline & & & & & & & $12 \%$ & \\
\hline & & & & & & & $18 \%$ & \\
\hline & & & & & & & $20 \%$ & \\
\hline & & & & & & & $\begin{array}{l}10 \% \\
12 \%\end{array}$ & \\
\hline & & & & & & & $\begin{array}{c}\left(1.88 \times 10^{9}\right. \\
\text { total } \\
\text { CFU/day })\end{array}$ & \\
\hline
\end{tabular}

\begin{tabular}{|c|c|c|c|c|c|c|c|c|}
\hline $\begin{array}{l}\text { Nikawa } \\
\text { et al. } \\
\text { (2004) [26] }\end{array}$ & $\begin{array}{l}\text { Double- } \\
\text { blind, } \\
\text { placebo- } \\
\text { controlled }\end{array}$ & $\begin{array}{l}\text { Female } \\
\text { dental } \\
\text { hygienist } \\
\text { students } \\
\text { (20 years } \\
\text { old) }\end{array}$ & Healthy & $\begin{array}{l}40 \text { subjects in } \\
\text { total }\end{array}$ & $\begin{array}{l}\text { yoghurt/day for } 2 \\
\text { weeks }+95 \mathrm{~g} \text { of } \\
\text { probiotic } \\
\text { yoghurt/day for } \\
2 \text { weeks } \\
95 \mathrm{~g} \text { of probiotic } \\
\text { yoghurt/day for } 2 \\
\text { weeks }+95 \mathrm{~g} \text { of } \\
\text { placebo yoghurt/day } \\
\text { for } 2 \text { weeks }\end{array}$ & $\begin{array}{c}\text { L. reuteri } \\
\text { SD2112 } \\
\text { (ATCC55730) }\end{array}$ & $\begin{array}{l}\text { Data not } \\
\text { provided }\end{array}$ & $\begin{array}{l}\text { Probiotic yoghurt } \\
\text { compared to the } \\
\text { placebo yoghurt } \\
\text { significantly reduced } \\
\text { MS counts. }\end{array}$ \\
\hline $\begin{array}{l}\text { Caglar } \\
\text { et al. } \\
\text { (2005) [47] }\end{array}$ & $\begin{array}{l}\text { Randomized, } \\
\text { double- } \\
\text { blind } \\
\text { crossover }\end{array}$ & $\begin{array}{c}\text { Young } \\
\text { adults } \\
(21-24 \\
\text { years old) }\end{array}$ & Healthy & $\begin{array}{c}\text { Test }(n=21) \\
\text { Control }(n=21)\end{array}$ & $\begin{array}{l}\text { Periods 1,3: run-in } \\
\text { and wash-out, } \\
\text { respectively } \\
\text { Periods } 2,4(2 \text { weeks } \\
\text { each): } 1 \times 200 \mathrm{~g} \text { of } \\
\text { probiotic or placebo } \\
\text { yoghurt/day }\end{array}$ & $\begin{array}{c}\text { Bifidobacterium } \\
\text { animalis lactis } \\
\text { DN-173 } 010\end{array}$ & $\begin{array}{l}7 \times 10^{7} \\
\mathrm{CFU} / \mathrm{g}\end{array}$ & $\begin{array}{l}\text { Significant decrease in } \\
\text { MS counts and a } \\
\text { tendency toward } \\
\text { Lactobacilli reduction } \\
\text { due to probiotic } \\
\text { yoghurt consumption. }\end{array}$ \\
\hline $\begin{array}{l}\text { Caglar } \\
\text { et al. } \\
\text { (2006) [27] }\end{array}$ & $\begin{array}{l}\text { Randomized, } \\
\text { placebo- } \\
\text { controlled } \\
\text { with } 4 \\
\text { parallel } \\
\text { arms }\end{array}$ & $\begin{array}{c}\text { Young } \\
\text { adults } \\
(21-24 \\
\text { years old) }\end{array}$ & Healthy & $\begin{array}{l}\text { Group A }(n=30) \\
\text { Group B }(n=30) \\
\text { Group C }(n=30) \\
\text { Group D }(n=30)\end{array}$ & $\begin{array}{l}200 \mathrm{~mL} \text { of water/day } \\
\text { through probiotic } \\
\text { straw for } 3 \text { weeks } \\
200 \mathrm{~mL} \text { of water/day } \\
\text { through placebo } \\
\text { straw for } 3 \text { weeks } \\
1 \text { probiotic tablet/day } \\
\text { for } 3 \text { weeks } \\
1 \text { placebo tablet/day } \\
\text { for } 3 \text { weeks }\end{array}$ & $\begin{array}{c}\text { L. reuteri } \\
\text { ATCC } 55730\end{array}$ & $\begin{array}{c}10^{8} \\
\mathrm{CFU} / \text { straw } \\
10^{8} \\
\mathrm{CFU} / \text { tablet }\end{array}$ & $\begin{array}{l}\text { Significant decrease in } \\
\text { MS counts and } \\
\text { tendency toward } \\
\text { reduction of Lactobacilli } \\
\text { scores in both probiotic } \\
\text { groups. }\end{array}$ \\
\hline $\begin{array}{l}\text { Caglar } \\
\text { et al. } \\
\text { (2008) [44] }\end{array}$ & $\begin{array}{l}\text { Randomized, } \\
\text { double- } \\
\text { blind, } \\
\text { placebo- } \\
\text { controlled } \\
\text { crossover }\end{array}$ & $\begin{array}{c}\text { Young in- } \\
\text { dividuals } \\
\text { (mean age } \\
20 \text { years } \\
\text { old) }\end{array}$ & Healthy & $\begin{array}{c}\text { Test }(n=23) \\
\text { Control }(n=24)\end{array}$ & $\begin{array}{l}\text { Periods 1,3: run-in } \\
\text { and wash-out, } \\
\text { respectively } \\
\text { Periods } 2,4 \text { (10 days } \\
\text { each): } 1 \times 53 \text { g of } \\
\text { probiotic or placebo } \\
\text { ice-cream/day }\end{array}$ & $\begin{array}{l}\text { B. animalis } \\
\text { lactis } \mathrm{Bb}-12\end{array}$ & $\begin{array}{l}1 \times 10^{7} \\
\mathrm{CFU} / \mathrm{g}\end{array}$ & $\begin{array}{l}\text { Significant decrease in } \\
\text { MS counts after } \\
\text { probiotic ice-cream } \\
\text { consumption. No } \\
\text { change in Lactobacilli } \\
\text { counts after both } \\
\text { ice-creams intake. }\end{array}$ \\
\hline
\end{tabular}


Table 2. Cont

\begin{tabular}{|c|c|c|c|c|c|c|c|c|}
\hline $\begin{array}{c}\text { Baseline } \\
\text { Condition }\end{array}$ & $\begin{array}{c}\text { Type of } \\
\text { Study }\end{array}$ & $\begin{array}{c}\text { Patient } \\
\text { Type }\end{array}$ & $\begin{array}{l}\text { Baseline } \\
\text { Condition }\end{array}$ & Study Groups & Treatment & $\begin{array}{l}\text { Probiotic } \\
\text { Strains }\end{array}$ & $\begin{array}{l}\text { Strain Con- } \\
\text { centration }\end{array}$ & Results \\
\hline $\begin{array}{l}\text { Stecksen- } \\
\text { Blicks } \\
\text { et al. } \\
\text { (2009) [25] }\end{array}$ & $\begin{array}{l}\text { Clustered, } \\
\text { double- } \\
\text { blind, } \\
\text { placebo- } \\
\text { controlled }\end{array}$ & $\begin{array}{l}\text { Preschool } \\
\text { children } \\
(1-5 \text { years } \\
\text { old })\end{array}$ & $\begin{array}{l}\text { Healthy } \\
\text { Caries }\end{array}$ & $\begin{array}{c}\text { Probiotic }(n= \\
110) \\
\text { Placebo }(n=76)\end{array}$ & $\begin{array}{c}1 \times 150 \mathrm{~mL} \text { probiotic } \\
\text { milk (supplemented } \\
\text { with } 2.5 \mathrm{mg} \\
\text { fluoride } / \mathrm{L} \text { ) } / \text { day for } \\
21 \mathrm{months} \\
1 \times 150 \mathrm{~mL} \text { standard } \\
\text { milk fluoride) } / \text { day } \\
\text { for } 21 \text { months }\end{array}$ & $\begin{array}{l}\text { L. rhamnosus } \\
\text { LB21 }\end{array}$ & $\begin{array}{c}1 \times 10^{7} \\
\mathrm{CFU} / \mathrm{mL}\end{array}$ & $\begin{array}{l}\text { Significant reduction in } \\
\text { caries development } \\
\text { after intervention milk } \\
\text { intake. Lower, but not } \\
\text { significant, MS } \\
\text { proportion and no } \\
\text { increase in total } \\
\text { Lactobacilli counts in } \\
\text { the probiotic group. }\end{array}$ \\
\hline $\begin{array}{c}\text { Singh } \\
\text { et al. } \\
\text { (2011) [40] }\end{array}$ & $\begin{array}{l}\text { Randomized, } \\
\text { double- } \\
\text { blind, } \\
\text { placebo- } \\
\text { controlled, } \\
\text { crossover }\end{array}$ & $\begin{array}{l}\text { Children } \\
(12-14 \\
\text { years old })\end{array}$ & Healthy & $\begin{array}{l}\text { Group I }(n=20) \\
\text { Group II }(n=20)\end{array}$ & $\begin{array}{c}\text { 1-week run-in, } 54 \mathrm{~g} \text { of } \\
\text { placebo } \\
\text { ice-cream/day for } \\
10 \text { days, } 2 \text {-week wash } \\
\text { out, } 54 \mathrm{~g} \text { of probiotic } \\
\text { ice-cream/day for } \\
10 \text { days } \\
\text { 1-week run-in, } 54 \mathrm{~g} \text { of } \\
\text { probiotic } \\
\text { ice-cream/day for } \\
10 \text { days, } 2 \text {-week wash } \\
\text { out, } 54 \mathrm{~g} \text { of placebo } \\
\text { ice-cream/day for } \\
10 \text { days }\end{array}$ & $\begin{array}{l}\text { B. animalis } \\
\text { lactis BB-12 } \\
\text { ATCC } 27536 \\
\text { L. acidophilus } \\
\text { La-5 }\end{array}$ & $\begin{array}{l}1 \times 10^{6} \\
\mathrm{CFU} / \mathrm{g} \\
1 \times 10^{6} \\
\mathrm{CFU} / \mathrm{g}\end{array}$ & $\begin{array}{l}\text { Significant decrease in } \\
\text { MS counts after } \\
\text { probiotic ice-cream } \\
\text { consumption. No } \\
\text { change in Lactobacilli } \\
\text { counts after both } \\
\text { ice-creams intake. }\end{array}$ \\
\hline $\begin{array}{l}\text { Chuang } \\
\text { et al. } \\
\text { (2011) [35] }\end{array}$ & $\begin{array}{l}\text { Randomized, } \\
\text { double- } \\
\text { blind, } \\
\text { placebo- } \\
\text { controlled }\end{array}$ & $\begin{array}{c}\text { Young } \\
\text { adults } \\
(20-26 \\
\text { years old })\end{array}$ & Healthy & $\begin{array}{c}\text { Test }(n=42) \\
\text { Control }(n=36)\end{array}$ & $\begin{array}{l}3 \text { probiotic }(+11 \% \\
\text { xylitol) tablets/day } \\
\text { for } 2 \text { weeks } \\
3 \text { placebo }(11 \% \\
\text { xylitol) tablets/day } \\
\text { for } 2 \text { weeks }\end{array}$ & $\begin{array}{l}\text { L. paracasei } \\
\text { GMNL-33 }\end{array}$ & $\begin{array}{c}3 \times 10^{8} \\
\text { cells/tablet }\end{array}$ & $\begin{array}{l}\text { No change in MS and } \\
\text { Lactobacilli levels in } \\
\text { both groups during the } \\
\text { experiment. Significant } \\
\text { reduction in MS counts } \\
\text { in the post-treatment } \\
\text { period compared to the } \\
\text { respective levels } \\
\text { recorded immediately } \\
\text { after treatment } \\
\text { cessation. }\end{array}$ \\
\hline $\begin{array}{l}\text { Kavaloglu- } \\
\text { Cildir } \\
\text { et al. } \\
\text { (2011) [29] }\end{array}$ & $\begin{array}{l}\text { Randomized, } \\
\text { double- } \\
\text { blind, } \\
\text { placebo- } \\
\text { controlled, } \\
\text { crossover }\end{array}$ & $\begin{array}{l}\text { Children } \\
\quad(4-12 \\
\text { years old })\end{array}$ & $\begin{array}{l}\text { Healthy } \\
\text { Cleft } \\
\text { Lip/Palate }\end{array}$ & $\begin{array}{c}\text { Test }(n=19) \\
\text { Control }(n=19)\end{array}$ & $\begin{array}{l}\text { Periods 1,3: run-in } \\
\text { and wash-out, } \\
\text { respectively } \\
\text { Periods } 2,4 \text { ( } 25 \text { days } \\
\text { each): } 5 \text { probiotics or } \\
\text { placebo drops/day }\end{array}$ & $\begin{array}{l}\text { L. reuteri DSM } \\
17938 \\
\text { L. reuteri } \\
\text { ATCC PTA } \\
5289\end{array}$ & $\begin{array}{l}\geq 1 \times 10^{8} \\
\text { CFU } / 5 \\
\text { drops } \\
\geq 1 \times 10^{8} \\
\text { CFU } / 5 \\
\text { drops }\end{array}$ & $\begin{array}{l}\text { No change in MS and } \\
\text { Lactobacilli counts after } \\
\text { the consumption of } \\
\text { both drops. }\end{array}$ \\
\hline $\begin{array}{c}\text { Juneja } \\
\text { et al. } \\
\text { (2012) [24] }\end{array}$ & $\begin{array}{l}\text { Randomized, } \\
\text { double- } \\
\text { blind, } \\
\text { placebo- } \\
\text { controlled }\end{array}$ & $\begin{array}{l}\text { Children } \\
(12-15 \\
\text { years old })\end{array}$ & $\begin{array}{l}\text { Healthy } \\
\text { Caries }\end{array}$ & $\begin{array}{l}\text { Group I }(n=18) \\
\text { Group II }(n=18)\end{array}$ & $\begin{array}{l}2 \times 150 \mathrm{~mL} \text { of } \\
\text { standard milk/day } \\
\text { for } 3 \text { weeks } \\
2 \times 150 \mathrm{~mL} \text { of } \\
\text { probiotic milk/day } \\
\text { for } 3 \text { weeks }\end{array}$ & $\begin{array}{l}\text { L. rhamnosus } \\
\text { het } 70\end{array}$ & $\begin{array}{l}2.34 \times 10^{9} \\
\text { CFU/day }\end{array}$ & $\begin{array}{l}\text { Significant reduction of } \\
\text { MS levels immediately } \\
\text { after the intake of } \\
\text { probiotic milk. }\end{array}$ \\
\hline $\begin{array}{l}\text { Burton } \\
\text { et al. } \\
\text { (2013) [54] }\end{array}$ & $\begin{array}{l}\text { Randomized, } \\
\text { double- } \\
\text { blind, } \\
\text { placebo- } \\
\text { controlled }\end{array}$ & $\begin{array}{l}\text { Schoolchildren } \\
\quad(5-10 \\
\text { years old })\end{array}$ & $\begin{array}{l}\text { Healthy } \\
\text { Caries }\end{array}$ & $\begin{array}{c}\text { Test }(n=40) \\
\text { Control }(n=43)\end{array}$ & $\begin{array}{c}2 \text { probiotic } \\
\text { lozenges/day for } \\
3 \text { months } \\
2 \text { placebo } \\
\text { lozenges/day for } \\
3 \text { months }\end{array}$ & $\begin{array}{c}\text { S. salivarius } \\
\text { M18 }\end{array}$ & $\begin{array}{l}3.6 \times 10^{9} \\
\text { CFU/lozenge }\end{array}$ & $\begin{array}{l}\text { Significant reduction of } \\
\text { plaque scores in the } \\
\text { probiotic group. } \\
\text { Children who presented } \\
\text { a distinct oral } \\
\text { colonization by M18 } \\
\text { tended to possess lower } \\
\text { counts of MS. }\end{array}$ \\
\hline $\begin{array}{l}\text { Teanpaisan } \\
\text { et al. } \\
\text { (2013) [36] }\end{array}$ & $\begin{array}{l}\text { Randomized, } \\
\text { double- } \\
\text { blind, } \\
\text { placebo- } \\
\text { controlled }\end{array}$ & $\begin{array}{c}\text { Young } \\
\text { adults } \\
(18-25 \\
\text { years old })\end{array}$ & $\begin{array}{l}\text { Healthy } \\
\text { Caries }\end{array}$ & $\begin{array}{l}\text { Group A }(n=20) \\
\text { Group B }(n=17)\end{array}$ & $\begin{array}{c}1 \times 10 \mathrm{~g} \text { reconstituted } \\
\text { probiotic milk } \\
\text { powder in } 50 \mathrm{~mL} \text { of } \\
\text { water } / \text { day for } \\
4 \text { weeks } \\
1 \times 10 \mathrm{~g} \text { reconstituted } \\
\text { placebo milk powder } \\
\text { in } 50 \mathrm{~mL} \text { of } \\
\text { water } / \text { day for } \\
4 \text { weeks }\end{array}$ & $\begin{array}{l}\text { L. paracasei } \\
\text { SD1 }\end{array}$ & $\begin{array}{l}\geq 10^{7} \\
\mathrm{CFU} / \mathrm{g} \text { or } \\
\mathrm{mL}\end{array}$ & $\begin{array}{l}\text { Significant decrease of } \\
\text { MS levels and increase } \\
\text { of Lactobacilli levels } \\
\text { after probiotic milk } \\
\text { powder consumption. } \\
\text { The probiotic could be } \\
\text { detected up to } 4 \text { weeks } \\
\text { after the } \\
\text { discontinuation of the } \\
\text { intervention. }\end{array}$ \\
\hline $\begin{array}{c}\text { Yadav } \\
\text { et al. } \\
\text { (2014) [33] }\end{array}$ & $\begin{array}{l}\text { Randomized, } \\
\text { double- } \\
\text { blind, } \\
\text { placebo- } \\
\text { controlled, } \\
\text { crossover }\end{array}$ & $\begin{array}{l}\text { Children } \\
\text { (6-8 years } \\
\text { old) }\end{array}$ & $\begin{array}{l}\text { Healthy } \\
\text { Caries }\end{array}$ & $\begin{array}{c}\text { Test }(n=31) \\
\text { Control }(n=31)\end{array}$ & $\begin{array}{c}\text { Periods 1,3: run-in } \\
\text { (7 days) and } \\
\text { wash-out ( } 30 \text { days), } \\
\text { respectively } \\
\text { Periods } 2,4(10 \text { days } \\
\text { each): } 1 \times 10 \mathrm{~mL} \text { of } \\
\text { probiotic or placebo } \\
\text { milk/day }\end{array}$ & L. casei Shirota & $\begin{array}{l}\text { Data not } \\
\text { provided }\end{array}$ & $\begin{array}{l}\text { Significant reduction of } \\
\text { MS counts after the } \\
\text { intake of probiotic milk. }\end{array}$ \\
\hline
\end{tabular}


Table 2. Cont

\begin{tabular}{|c|c|c|c|c|c|c|c|c|}
\hline $\begin{array}{l}\text { Baseline } \\
\text { Condition }\end{array}$ & $\begin{array}{l}\text { Type of } \\
\text { Study }\end{array}$ & $\begin{array}{l}\text { Patient } \\
\text { Type }\end{array}$ & $\begin{array}{l}\text { Baseline } \\
\text { Condition }\end{array}$ & Study Groups & Treatment & $\begin{array}{l}\text { Probiotic } \\
\text { Strains }\end{array}$ & $\begin{array}{l}\text { Strain Con- } \\
\text { centration }\end{array}$ & Results \\
\hline $\begin{array}{l}\text { Pinto et al. } \\
\text { (2014) [48] }\end{array}$ & $\begin{array}{l}\text { Randomized, } \\
\text { double- } \\
\text { blind, } \\
\text { placebo- } \\
\text { controlled, } \\
\text { crossover }\end{array}$ & $\begin{array}{l}\text { Orthodontic } \\
\text { patients } \\
\text { (median } \\
\text { age } 15 \\
\text { years) }\end{array}$ & Healthy & $\begin{array}{l}\text { Group } 1(n=15) \\
\text { Group } 2(n=15)\end{array}$ & $\begin{array}{c}\text { 1-week run-in, } 200 \mathrm{~g} \\
\text { of probiotic } \\
\text { yoghurt/day for } \\
2 \text { weeks, } 4 \text {-week } \\
\text { wash out, } 1 \times 200 \mathrm{~g} \text { of } \\
\text { placebo yoghurt/day } \\
\text { for } 2 \text { weeks } \\
\text { 1-week run-in, } 200 \mathrm{~g} \\
\text { of placebo } \\
\text { yoghurt/day for } \\
2 \text { weeks, 4-week } \\
\text { wash out, } 200 \mathrm{~g} \text { of } \\
\text { placebo } \\
\text { ice-cream/day for } \\
2 \text { weeks }\end{array}$ & $\begin{array}{l}\text { B. animalis } \\
\text { lactis } \\
\text { DN-173010 }\end{array}$ & $\begin{array}{l}\text { Data not } \\
\text { provided }\end{array}$ & $\begin{array}{c}\text { No significant } \\
\text { difference in MS, } \\
\text { Lactobacilli and total } \\
\text { cultivable } \\
\text { microorganisms counts } \\
\text { after both yoghurts. } \\
\text { Both yoghurts were } \\
\text { equally efficient at } \\
\text { reducing total cultivable } \\
\text { microorganisms } \\
\text { isolated from } \\
\text { dental plaque. }\end{array}$ \\
\hline $\begin{array}{l}\text { Nishihara } \\
\text { et al. } \\
\text { (2014) [41] }\end{array}$ & $\begin{array}{l}\text { Randomized, } \\
\text { double- } \\
\text { blind, } \\
\text { placebo- } \\
\text { controlled } \\
\text { with } 4 \\
\text { parallel } \\
\text { arms } \\
\text { +Cohort } \\
\text { study }\end{array}$ & $\begin{array}{l}\text { Sixth-year } \\
\text { dental } \\
\text { students } \\
\text { (mean age } \\
24.8 \\
\text { years) } \\
\\
\text { Dentists } \\
\text { (mean age } \\
30.0 \\
\text { years) }\end{array}$ & $\begin{array}{l}\text { Healthy } \\
\text { Healthy }\end{array}$ & $\begin{array}{l}\text { Group } 1(n=17) \\
\text { Group } 2(n=16) \\
\text { Group } 3(n=13) \\
\text { Group } 4(n=18) \\
1 \text { test group }(n= \\
8)\end{array}$ & $\begin{array}{c}1 \text { probiotic }(+280 \mathrm{mg} \\
\text { xylitol) tablet for } \\
1 \mathrm{month} \\
1 \text { probiotic }(+450 \mathrm{mg} \\
\text { xylitol) tablet for } \\
1 \mathrm{month} \\
1 \text { Ovalgen }(+100 \mathrm{mg} \\
\text { xylitol) tablet for } \\
1 \text { month } \\
1 \text { xylitol ( } 280 \mathrm{mg}) \\
\text { tablet for } 1 \mathrm{month} \\
3 \times 1 \text { tablet/day for } \\
2 \text { weeks }\end{array}$ & $\begin{array}{l}\text { L. salivarius } \\
\text { WB21 } \\
\text { L. salivarius TI } \\
2711 \\
\text { L. salivarius } \\
\text { WB21 }\end{array}$ & $\begin{array}{c}6.7 \times 10^{8} \\
\text { CFU/tablet } \\
2.8 \times 10^{8} \\
\text { CFU/tablet } \\
\\
2.0 \times 10^{9} \\
\mathrm{CFU} / \text { tablet }\end{array}$ & $\begin{array}{l}\text { No significant change in } \\
\text { MS levels. Significant } \\
\text { increase in Lactobacilli } \\
\text { counts in the two } \\
\text { probiotic groups and } \\
\text { enhanced buffering } \\
\text { capacity in L. salivarius } \\
\text { TI } 2711 \text { and Ovalgen } \\
\text { group. } \\
\text { Significant decrease in } \\
\text { salivary MS levels. }\end{array}$ \\
\hline $\begin{array}{c}\text { Keller } \\
\text { et al. } \\
\text { (2014) [30] }\end{array}$ & $\begin{array}{l}\text { Randomized, } \\
\text { double- } \\
\text { blind, } \\
\text { placebo- } \\
\text { controlled }\end{array}$ & $\begin{array}{l}\text { Adolescents } \\
\quad(12-17 \\
\text { years old })\end{array}$ & $\begin{array}{l}\text { Healthy } \\
\text { Caries }\end{array}$ & $\begin{array}{c}\text { Test }(n=19) \\
\text { Control }(n=17)\end{array}$ & $\begin{array}{c}2 \text { probiotic } \\
\text { tablets/day for } \\
12 \text { weeks } \\
2 \text { placebo tablets/day } \\
\text { for } 12 \text { weeks }\end{array}$ & $\begin{array}{l}\text { L. reuteri DSM } \\
17938 \\
\text { L. reuteri } \\
\text { ATCC PTA } \\
5289\end{array}$ & $\begin{array}{c}1 \times 10^{8} \\
\mathrm{CFU} / \text { tablet } \\
1 \times 10^{8} \\
\mathrm{CFU} / \text { tablet }\end{array}$ & $\begin{array}{l}\text { Significant decrease of } \\
\text { fluorescence in decayed } \\
\text { teeth over time in the } \\
\text { probiotic group. No } \\
\text { significant differences } \\
\text { in fluorescence between } \\
\text { the two groups. }\end{array}$ \\
\hline $\begin{array}{c}\text { Gizani } \\
\text { et al. } \\
\text { (2016) [31] }\end{array}$ & $\begin{array}{l}\text { Randomized, } \\
\text { double- } \\
\text { blind, } \\
\text { placebo- } \\
\text { controlled }\end{array}$ & $\begin{array}{l}\text { Adolescents } \\
\text { and } \\
\text { young } \\
\text { adults } \\
\text { (mean age } \\
15.9 \\
\text { years) }\end{array}$ & $\begin{array}{l}\text { Healthy } \\
\text { Orthodontic } \\
\text { treatment } \\
\text { Caries }\end{array}$ & $\begin{array}{c}\text { Test }(n=42) \\
\text { Control }(n=43)\end{array}$ & $\begin{array}{c}1 \text { probiotic } \\
\text { lozenge/day for } \\
17 \text { months } \\
1 \text { placebo } \\
\text { lozenge/day for } \\
17 \text { months }\end{array}$ & $\begin{array}{l}\text { L. reuteri DSM } \\
17938 \\
\text { L. reuteri } \\
\text { ATCC PTA } \\
5289\end{array}$ & $\begin{array}{c}\geq 10^{8} \\
\text { CFU } / \text { lozenge } \\
\geq 10^{8} \\
\text { CFU } / \text { lozenge }\end{array}$ & $\begin{array}{l}\text { Significant reduction of } \\
\text { Lactobacilli counts and } \\
\text { no alteration of MS } \\
\text { levels in both groups. } \\
\text { No difference in the } \\
\text { incidence of white spot } \\
\text { lesions between the } \\
\text { two groups. }\end{array}$ \\
\hline $\begin{array}{l}\text { Ghasemi } \\
\text { et al. } \\
\text { (2017) [39] }\end{array}$ & $\begin{array}{l}\text { Randomized, } \\
\text { double- } \\
\text { blind, } \\
\text { placebo- } \\
\text { controlled }\end{array}$ & $\begin{array}{c}\text { Female } \\
\text { students } \\
(19-27 \\
\text { years old })\end{array}$ & Healthy & $\begin{array}{l}\text { Group } 1(n=25) \\
\text { Group } 2(n=25)\end{array}$ & $\begin{array}{c}200 \mathrm{~g} \text { of probiotic } \\
\text { yoghurt/day for } \\
3 \text { weeks } \\
3 \times 2 \text { xylitol } \\
\text { gums / day for } \\
3 \text { weeks }\end{array}$ & $\begin{array}{l}\text { L. acidophilus } \\
\text { ATCC } 4356 \\
\text { B. bifidum } \\
\text { ATCC } 29521\end{array}$ & $\begin{array}{c}1.5 \times 10^{8} \\
\text { total CFU/g }\end{array}$ & $\begin{array}{l}\text { Significant reduction of } \\
\text { MS counts in both } \\
\text { groups with no } \\
\text { significant difference } \\
\text { between them. }\end{array}$ \\
\hline $\begin{array}{l}\text { Koopaie } \\
\text { et al. } \\
\text { (2019) [55] }\end{array}$ & $\begin{array}{l}\text { Randomized, } \\
\text { double- } \\
\text { blind, } \\
\text { placebo- } \\
\text { controlled, } \\
\text { crossover }\end{array}$ & $\begin{array}{l}\text { Adolescents } \\
\text { and } \\
\text { adults } \\
\text { (mean age } \\
41.67 \\
\text { years) }\end{array}$ & Healthy & $\begin{array}{l}\text { Group } 1(n=20) \\
\text { Group } 2(n=20)\end{array}$ & $\begin{array}{c}70 \mathrm{~g} \text { of probiotic } \\
\text { cake/day for } 1 \text { week, } \\
\text { 4-week wash-out } \\
\text { period, } 70 \mathrm{~g} \text { of regular } \\
\text { cake/day for } 1 \text { week } \\
70 \mathrm{~g} \text { of regular } \\
\text { cake/day for } 1 \text { week, } \\
\text { 4-week wash-out } \\
\text { period, } 70 \mathrm{~g} \text { of } \\
\text { probiotic cake/day } \\
\text { for } 1 \text { week }\end{array}$ & B. coagulans & $\begin{array}{l}\text { Data not } \\
\text { provided }\end{array}$ & $\begin{array}{l}\text { No statistical difference } \\
\text { in MS levels after } \\
\text { probiotic cake intake. } \\
\text { Significant increase of } \\
\text { MS counts after regular } \\
\text { cake consumption. No } \\
\text { significant alteration in } \\
\text { salivary pH after the } \\
\text { consumption of } \\
\text { both cakes. }\end{array}$ \\
\hline $\begin{array}{l}\text { Javid et al. } \\
(2020) \text { [46] }\end{array}$ & $\begin{array}{l}\text { Randomized, } \\
\text { double- } \\
\text { blind, } \\
\text { placebo- } \\
\text { controlled }\end{array}$ & $\begin{array}{l}\text { Students } \\
(18-30 \\
\text { years old })\end{array}$ & $\begin{array}{l}\text { Healthy } \\
\text { Caries }\end{array}$ & $\begin{array}{c}\text { Test }(n=33) \\
\text { Control }(n=33)\end{array}$ & $\begin{array}{c}300 \mathrm{~g} \text { of probiotic } \\
\text { yoghurt/day for } \\
2 \text { weeks } \\
300 \mathrm{~g} \text { of placebo } \\
\text { yoghurt/day for } \\
2 \text { weeks }\end{array}$ & B. lactis $\mathrm{Bb}-12$ & $10^{6} \mathrm{CFU} / \mathrm{ml}$ & $\begin{array}{l}\text { Significant reduction in } \\
\text { MS and Lactobacilli } \\
\text { levels in the } \\
\text { probiotic group. }\end{array}$ \\
\hline $\begin{array}{c}\text { Ferrer } \\
\text { et al. } \\
(2020)[53]\end{array}$ & $\begin{array}{l}\text { Prospective, } \\
\text { mechanistic } \\
\text { pilot with } \\
\text { two parallel } \\
\text { follow-up } \\
\text { groups }\end{array}$ & $\begin{array}{c}\text { Adults } \\
(25-35 \\
\text { years old })\end{array}$ & Healthy & $\begin{array}{l}\text { Group } 1(n=6) \\
\text { Group } 2(n=5)\end{array}$ & $\begin{array}{l}7 \text { vials (multidose) } \\
\text { containing the } \\
\text { probiotic strain } \\
2 \text { vial (monodose) } \\
\text { containing the } \\
\text { probiotic strain }\end{array}$ & $\begin{array}{l}\text { S. dentisani } \\
\text { CECT7746 }\end{array}$ & $\begin{array}{c}5.5 \times 10^{9} \\
\text { CFU/vial } \\
4 \times 10^{10} \\
\text { CFU/vial }\end{array}$ & $\begin{array}{l}\text { Significant decrease of } \\
\text { MS and significant } \\
\text { increase in S. dentisani } \\
\text { levels and salivary pH. } \\
\text { The latter was stronger } \\
\text { in the } \\
\text { multi-dose schedule. }\end{array}$ \\
\hline
\end{tabular}


To cover the lack of knowledge about L. reuteri's effect on Lactobacilli salivary counts in humans, after the introduction of the strain Bifidobacterium DN-173 010 to the oral probiotic armamentarium [47], Caglar et al. selected L. reuteri ATCC 55,730 as intervention strain to illuminate this unknown aspect [27]. As concluded, probiotic-containing straws and tablets could be beneficial to $S$. mutans confinement. Two years later, Caglar and his affiliates presented the strain Bifidobacterium lactis Bb-12 as an anticaries-competent probiotic strain [44]. This study introduced a novel probiotic strain into the race against caries and suggested ice-cream as a possible means for probiotics administration.

Given the previous studies about probiotics' role in caries management, StecksenBlicks et al., came up with the highly promising idea of combining probiotic bacteria with fluoride [25]. This idea was based on the hypothesis that these two agents would act synergistically. Children in both the probiotic and placebo group consumed $150 \mathrm{~mL}$ of medium-fat milk at lunch for 21 months. As the results indicated, caries incidence increment was statistically significantly lower in the intervention group than in the control children. In contrast, salivary counts of caries-associated S. mutans and Lactobacilli were not affected.

With time passing by, innovative probiotic strains have been proposed for the fight against dental caries. For instance, L. acidophilus La5 combined with B. lactis Bb-12 [40], L. paracasei GMNL-33 [35], L. rhamnosus hct 70 [24], S. salivarius M18 [54], L. paracasei SD1 [36], L. casei Shirota [33], L. acidophilus ATCC 4356 combined with B. bifidum ATCC 29,521 [39] dismiss S. mutans from the oral cavity. Moreover, L. paracasei SD1, when being received once a day for 4 weeks can be retained in the oral cavity of healthy young adults for 4 additional weeks by the time the regimen has been interrupted [36]. Lately, a shift towards the study of the host-specific alterations caused by probiotics has been recorded. Remarkably, the potential of specific probiotics to decrease caries risk has been correlated with their property to increase saliva's buffering capacity [41,53], although no probiotic tested for an immediate anticaries ability has been shown to invert early caries development per se $[30,31]$.

It is worth mentioning that in 2019, a highly promising study was conducted [55]. This trial investigated the effect of a Bacillus coagulans-abundant cake on S. mutans levels and salivary $\mathrm{pH}$. It pointed out that the sweetened probiotic cake can keep $S$. mutans amounts low and comparable to those surveyed before cake consumption. Hence, it was proposed that cakes carrying probiotic flora may comprise a novel strategy against S. mutans [92] This proposal is the modern trend in food policy [93].

\section{Clinical Considerations on Probiotics' Effectiveness}

In general, for a strain to serve as a probiotic, it should be capable of firmly attaching to the oral surfaces [94]. However, Lactobacilli, present weak adhesiveness on the tooth structure [95]. The latter raises a variety of speculations around their long-term restraint in retention sites. Data from research studying the effect of probiotics on their saliva concentration and their tooth structure content are limited. However, according to Meurman et al., during the second week after the discontinuation of probiotic treatment with a yoghurt supplemented with LGG, LGG's salivary counts show a decrease in subjects who were following the treatment [20].

Likewise, Busscher et al. investigated LGG, L. acidophilus and B. bifidum's ability of adhesiveness on the tooth structure in vitro and in vivo [96]. In vitro data suggested that LGG possesses a by far inferior ability of adhesion to the clear enamel (without salivary pellicle) and the pellicle-coated enamel compared to the corresponding ability L. acidophilus. This difference was attributed to the hydrophilic character of LGG. Salivary samples collected from individuals who were subjected to the daily intake of these bacteria through a bio-yoghurt were free of Lactobacilli. It was concluded that the ecological conditions in the oral cavity of test persons were unfavorable for Lactobacilli to grow, as temporary colonization could not be achieved even in individuals without any evincible amounts of Lactobacilli. 
Another study demonstrating the temporary colonization of the oral cavity by probiotic Lactobacilli is Petti et al. [97]. The authors investigated if S. thermophilus and L. bulgaricus-containing yoghurt presented any activity against the oral microbiome regarding whether these probiotics could colonize the human oral cavity. Some activity against oral Streptococci was detected, but this has not resulted from the probiotics' colonization because it elapsed once the treatment was discontinued.

Yli-Knuuttila et al., except for demonstrating LGG's inability to colonize young adults' oral cavity, signalized that permanent LGG colonization is possible, providing that early administration in childhood has taken place [98]. Devine and Marsh [99] explained that the latter finding was correlated with the instability of the resident microbiota in children [100]. After that, many studies were conducted to examine the possible long-term effect of probiotics in the child population. These trials are synopsized in Table 3.

One study mentioned above evaluated the possible correlation between probiotic use in combination with an agent controlling the oral microbiota [23]. Aminabadi et al. tested this eventuality by combining the salutary advantages of chlorhexidine ( $\mathrm{CHX})$ in oral microbiome control using LGG. They concluded that CHX increases-at least 5 weeks after ceasing the regimen-the stability of LGG oral colonization.

Taipale et al. evaluated the influence of B. animalis lactis Bb-12 (Bb-12) early administration on S. mutans and Bb-12 oral colonization [45]. Subjects 1-2 months old in the test group received tablets with the probiotic strain, whereas control groups consumed xylitol $(\mathrm{X})$ and sorbitol (S) in the same manner. The whole regimen lasted until the infants became 2 years old. Qualitative PCR showed bare and no Bb-12 oral colonization at 8-month-old and 2-year-old children, respectively, significantly reduced S. mutans levels, and had no effect on Lactobacilli. Such results were extracted in a study carried out one year later for the strain L. paracasei F19 [34].

In 2014, Stensson and his team studied whether caries prevalence could be subverted through oral probiotic administration before establishing the oral microflora [28]. It is worth mentioning that although a significant reduction in caries incidence was reported, no caries-associated microbiological alterations were observed. Other strains of L. reuteri (DSM 17,938 and ATCC PTA 5289), when administered through lozenges twice a day, prevail against MS and Lactobacilli and benefit the salivary buffer capacity [32].

Hedayati-Hajikand et al., in turn, evaluated the effect of a commercially known probiotic product (ProBiora3 TM) filled with the strains S. uberis $\mathrm{KJ} 2$, S. oralis $\mathrm{KJ} 3$, S. rattus JH145 as an adjunct to the everyday oral hygiene of 2/3-year-old children [52]. Thus, ProBiora3 TM chewing tablets benefit early childhood caries increment when used in children's daily oral care.

More recent studies suggest that L. rhamnosus combined with B. longum, L. paracasei $\mathrm{SD} 1$, and L. brevis CD2 could also assist in attenuating the range of dental caries from early ages $[37,38,42,49]$. More specifically, L. rhamnosus combined with $B$. longum, despite being incapable of reducing S. mutans salivary levels, they do so as far as Lactobacilli are concerned and enhance the buffering capacity of saliva [49]. On the other hand, L. paracasei SD1 affects MS levels and inhibits caries development [37,38]. Finally, the latest research in the field suggests that $L$. brevis $\mathrm{CD} 2$ is competent for diabetic children because it improves some caries risk factors (e.g., reduction in salivary MS and maximum plaque $\mathrm{pH}$ fall, increase in lowest plaque $\mathrm{pH}$ ) and gingival health [42]. 
Table 3. Clinical trials investigating probiotics ability to colonize the oral cavity of children preemptively.

\begin{tabular}{|c|c|c|c|c|c|c|c|c|}
\hline $\begin{array}{l}\text { Baseline } \\
\text { Condition }\end{array}$ & Type of Study & $\begin{array}{l}\text { Patient } \\
\text { Type }\end{array}$ & $\begin{array}{l}\text { Baseline } \\
\text { Condition }\end{array}$ & Study Groups & Treatment & $\begin{array}{l}\text { Probiotic } \\
\text { Strains }\end{array}$ & $\begin{array}{l}\text { Strain } \\
\text { Concentra- } \\
\text { tion }\end{array}$ & Results \\
\hline $\begin{array}{l}\text { Aminabadi } \\
\text { et al. } \\
\text { (2011) [23] }\end{array}$ & $\begin{array}{l}\text { Randomized, } \\
\text { double-blind } \\
\text { with } 4 \text { parallel } \\
\text { arms }\end{array}$ & $\begin{array}{l}\text { Children } \\
(6-12 \\
\text { years old })\end{array}$ & Healthy & $\begin{array}{l}\text { Group A }(n=35) \\
\text { Group B }(n=35) \\
\text { Group C }(n=35)\end{array}$ & $\begin{array}{c}2 \times 5 \mathrm{~mL} \text { of } 0.12 \% \\
\text { chlorhexidine/day } \\
\text { for } 2 \text { weeks } \\
15-20 \mathrm{~mL} \text { of probiotic } \\
\text { yoghurt for } 3 \text { weeks } \\
2 \times 5 \mathrm{~mL} \text { of } 0.12 \% \\
\text { chlorhexidine/day } \\
\text { for } 2 \text { weeks } \\
+ \\
15-20 \text { mL of probiotic } \\
\text { yoghurt for } 3 \text { weeks }\end{array}$ & $\begin{array}{l}\text { L. rhamnosus } \\
\text { GG }\end{array}$ & $\begin{array}{l}2 \times 10^{8} \\
\mathrm{CFU} / \mathrm{g}\end{array}$ & $\begin{array}{l}\text { Significant decrease } \\
\text { in MS counts in all } \\
\text { groups; only in } \\
\text { groups A and C it } \\
\text { was persisted for } 5 \\
\text { weeks after the end of } \\
\text { treatment. In group C } \\
\text { LGG levels were } \\
\text { more prominent than } \\
\text { in group B. }\end{array}$ \\
\hline $\begin{array}{l}\text { Taipale } \\
\text { et al. } \\
\text { (2012) [45] }\end{array}$ & $\begin{array}{l}\text { Randomized, } \\
\text { double-blind, } \\
\text { placebo- } \\
\text { controlled } \\
\text { with } 3 \text { parallel } \\
\text { arms }\end{array}$ & $\begin{array}{l}\text { Infants (1-2 } \\
\text { months old) }\end{array}$ & Healthy & $\begin{array}{c}\text { Test }(n=32) \\
\text { Control } 1(n=35) \\
\text { Control } 2(n=29)\end{array}$ & $\begin{array}{c}2 \text { probiotic- } \\
\text { tablets/day } \\
2 \text { xylitol-tablets/day } \\
2 \text { sorbitol-tablets/day } \\
\text { Until the age of } \\
2 \text { years old }\end{array}$ & $\begin{array}{l}\text { B. animalis } \\
\text { lactis } \mathrm{BB}-12\end{array}$ & $\begin{array}{c}5 \times 10^{9} \\
\text { CFU/tablet }\end{array}$ & $\begin{array}{l}\text { Significant decrease } \\
\text { in MS counts in the } \\
\text { probiotic and the } \\
\text { sorbitol groups at the } \\
\text { age of } 2 \text { years. No } \\
\text { observed permanent } \\
\text { oral colonization of } \\
\text { BB-12. Lactobacilli } \\
\text { were unaffected. }\end{array}$ \\
\hline $\begin{array}{l}\text { Hasslöf } \\
\text { et al. } \\
\text { (2013) [34] }\end{array}$ & $\begin{array}{l}\text { Randomized, } \\
\text { double-blind, } \\
\text { placebo- } \\
\text { controlled }\end{array}$ & $\begin{array}{c}\text { Infants (4 } \\
\text { months old) }\end{array}$ & Healthy & $\begin{array}{c}\text { Test }(n=56) \\
\text { Control }(n=62)\end{array}$ & $\begin{array}{l}\text { At least } 1 \text { serving of } \\
\text { probiotic-cereals/day } \\
\text { At least } 1 \text { serving of } \\
\text { placebo-cereals/day }\end{array}$ & $\begin{array}{l}\text { L. paracasei } \\
\text { F19 }\end{array}$ & $\begin{array}{c}1 \times 10^{8} \\
\text { CFU/serving }\end{array}$ & $\begin{array}{l}\text { No significant } \\
\text { difference in MS } \\
\text { counts and caries } \\
\text { experience between } \\
\text { the two groups. No } \\
\text { permanent } \\
\text { establishment } \\
\text { of LF19. }\end{array}$ \\
\hline $\begin{array}{l}\text { Stensson } \\
\text { et al. } \\
\text { (2014) [28] }\end{array}$ & $\begin{array}{l}\text { Randomized, } \\
\text { single-blind, } \\
\text { placebo- } \\
\text { controlled }\end{array}$ & $\begin{array}{c}\text { Mothers } \\
\text { (during the } \\
\text { last month } \\
\text { of gestation) } \\
+ \\
\text { Infants } \\
\text { (through the } \\
\text { 1st year of } \\
\text { life) }\end{array}$ & Healthy & $\begin{array}{c}\text { Test }(n=60) \\
\text { Control }(n=53)\end{array}$ & $\begin{array}{c}5 \text { drop of } \\
\text { probiotic-oil/day } \\
\text { (last month of } \\
\text { gestation and 1st year } \\
\text { of life) } \\
5 \text { drops of } \\
\text { placebo-oil/day (last } \\
\text { month of gestation } \\
\text { and 1st year of life) }\end{array}$ & $\begin{array}{c}\text { L. reuteri } \\
\text { ATCC } 55730\end{array}$ & $\begin{array}{l}10^{8} \mathrm{CFU} / 5 \\
\text { drops }\end{array}$ & $\begin{array}{c}\text { Significant decrease } \\
\text { in caries prevalence } \\
\text { in the probiotic group. } \\
\text { No significant } \\
\text { intergroup } \\
\text { differences in } L . \\
\text { reuteri, MS, } \\
\text { Lactobacilli and } \\
\text { sIgA counts. }\end{array}$ \\
\hline $\begin{array}{l}\text { Hedayati- } \\
\text { Hajikand } \\
\text { et al. } \\
\text { (2015) [52] }\end{array}$ & $\begin{array}{l}\text { Randomized, } \\
\text { double-blind, } \\
\text { placebo- } \\
\text { controlled }\end{array}$ & $\begin{array}{l}\text { Preschool } \\
\text { children } \\
\quad(2-3 \\
\text { years old })\end{array}$ & $\begin{array}{l}\text { Healthy } \\
\text { Caries }\end{array}$ & $\begin{array}{c}\text { Test }(n=54) \\
\text { Control }(n=56)\end{array}$ & $\begin{array}{c}1 \text { chewing } \\
\text { probiotic-tablet/day } \\
1 \text { chewing } \\
\text { placebo-tablet/day }\end{array}$ & $\begin{array}{l}\text { S. uberis KJ2 } \\
\mathrm{TM} \\
\text { S. oralis } \mathrm{KJ} 3 \\
\mathrm{TM} \\
\text { S. rattus } \\
\text { JH145 }\end{array}$ & $\begin{array}{l}\geq 1 \times 10^{8} \\
\text { total CFU/ } \\
\quad \text { tablet }\end{array}$ & $\begin{array}{l}\text { Significantly lower } \\
\text { caries increment in } \\
\text { the probiotic group. }\end{array}$ \\
\hline $\begin{array}{l}\text { Villavicencio } \\
\text { et al. } \\
\text { (2017) [49] }\end{array}$ & $\begin{array}{l}\text { Randomized, } \\
\text { triple-blind, } \\
\text { placebo- } \\
\text { controlled }\end{array}$ & $\begin{array}{c}\text { Preschool } \\
\text { children } \\
(3-4 \\
\text { months old })\end{array}$ & $\begin{array}{l}\text { Healthy } \\
\text { Caries }\end{array}$ & $\begin{array}{c}\text { Test }(n=136) \\
\text { Control }(n=227)\end{array}$ & $\begin{array}{c}200 \mathrm{~mL} \text { of } \\
\text { reconstituted } \\
\text { probiotic milk/day } \\
\text { for } 5 \text { days a week } \\
\text { during a 9-month } \\
\text { period } \\
200 \mathrm{~mL} \text { of } \\
\text { reconstituted } \\
\text { standard } \\
\text { reconstituted } \\
\text { milk/day for } 5 \text { days a } \\
\text { week during a } \\
\text { 9-month period }\end{array}$ & $\begin{array}{l}\text { L. rhamnosus } \\
\text { B. longum }\end{array}$ & $\begin{array}{c}5 \times 10^{6} \\
\text { CFU/g of } \\
\text { powdered } \\
\text { milk } \\
3 \times 10^{6} \\
\text { CFU/g of } \\
\text { powdered } \\
\text { milk }\end{array}$ & $\begin{array}{l}\text { Significantly lower } \\
\text { counts of Lactobacilli } \\
\text { count and higher } \\
\text { buffering capacity in } \\
\text { the test group. No } \\
\text { significant difference } \\
\text { in caries prevalence, } \\
\text { MS counts, salivary } \\
\text { pH and dental plaque } \\
\text { between the groups. }\end{array}$ \\
\hline $\begin{array}{l}\text { Pahum- } \\
\text { unto et al. } \\
\text { (2018) [37] }\end{array}$ & $\begin{array}{l}\text { Randomized, } \\
\text { double-blind, } \\
\text { placebo- } \\
\text { controlled }\end{array}$ & $\begin{array}{l}\text { Preschool } \\
\text { children } \\
(1.5-5 \\
\text { years old })\end{array}$ & Healthy & $\begin{array}{c}\text { Test }(n=62) \\
\text { Control }(n=62)\end{array}$ & $\begin{array}{l}5 \mathrm{~g} \text { of probiotic milk } \\
\text { powder in } 50 \mathrm{~mL} \text { of } \\
\text { water/day for } \\
3 \text { months } \\
5 \mathrm{~g} \text { of standard milk } \\
\text { powder in } 50 \mathrm{~mL} \text { of } \\
\text { water/day for } \\
3 \text { months }\end{array}$ & $\begin{array}{l}\text { L. paracasei } \\
\text { SD1 }\end{array}$ & $\begin{array}{l}1 \times 10^{7} \\
\mathrm{CFU} / \mathrm{g}\end{array}$ & $\begin{array}{l}\text { Significantly lower } \\
\text { risk of MS levels } \\
\text { increases and of } \\
\text { caries development } \\
\text { in the test group. }\end{array}$ \\
\hline $\begin{array}{l}\text { Alamoudi } \\
\text { et al. } \\
\text { (2018) [32] }\end{array}$ & $\begin{array}{l}\text { Randomized, } \\
\text { double-blind, } \\
\text { placebo- } \\
\text { controlled }\end{array}$ & $\begin{array}{l}\text { Children } \\
\quad(3-6 \\
\text { years old })\end{array}$ & Healthy & $\begin{array}{c}\text { Test }(n=90) \\
\text { Control }(n=88)\end{array}$ & $\begin{array}{c}2 \text { probiotic } \\
\text { lozenges/day for } \\
28 \text { days } \\
2 \text { placebo } \\
\text { lozenges/day for } \\
28 \text { days }\end{array}$ & $\begin{array}{l}\text { L. reuteri } \\
\text { DSM } 17938 \\
\text { L. reuteri } \\
\text { ATCC PTA } \\
5289\end{array}$ & $\begin{array}{c}\geq 2 \times 10^{8} \\
\text { total } \\
\text { CFU/lozenge }\end{array}$ & $\begin{array}{c}\text { Significant decrease } \\
\text { in MS and } \\
\text { Lactobacilli counts in } \\
\text { the probiotic group. } \\
\text { No statistical } \\
\text { difference in plaque } \\
\text { accumulation and } \\
\text { buffer capacity } \\
\text { between the groups. }\end{array}$ \\
\hline
\end{tabular}


Table 3. Cont

\begin{tabular}{|c|c|c|c|c|c|c|c|c|}
\hline $\begin{array}{l}\text { Baseline } \\
\text { Condition }\end{array}$ & Type of Study & $\begin{array}{l}\text { Patient } \\
\text { Type }\end{array}$ & $\begin{array}{l}\text { Baseline } \\
\text { Condition }\end{array}$ & Study Groups & Treatment & $\begin{array}{c}\text { Probiotic } \\
\text { Strains }\end{array}$ & $\begin{array}{l}\text { Strain } \\
\text { Concentra- } \\
\text { tion }\end{array}$ & Results \\
\hline $\begin{array}{l}\text { Manmon- } \\
\text { tri et al. } \\
(2020)[38]\end{array}$ & $\begin{array}{c}\text { Randomized, } \\
\text { double-blind, } \\
\text { placebo- } \\
\text { controlled } \\
\text { with } 3 \\
\text { parallel arms }\end{array}$ & $\begin{array}{l}\text { Preschool } \\
\text { children } \\
\quad(1-5 \\
\text { years old })\end{array}$ & $\begin{array}{l}\text { Healthy } \\
\text { Caries }\end{array}$ & $\begin{array}{l}\text { Group I }(n=86) \\
\text { Group II }(n=89) \\
\text { Group III }(n=93)\end{array}$ & $\begin{array}{c}1 \times 3 \text { g of placebo } \\
\text { milk powder in } \\
50 \text { mL of milk for } \\
7 \text { days / week for } \\
6 \text { months } \\
1 \times 3 \text { g of probiotic } \\
\text { milk powder in } \\
50 \text { mL of milk for } \\
7 \text { days / week for } \\
6 \text { months } \\
1 \times 3 \text { g of probiotic } \\
\text { milk powder in } \\
50 \text { mL of milk for } \\
3 \text { days } / \text { week }+3 \text { g of } \\
\text { placebo milk powder } \\
\text { in } 50 \text { mL of milk for } \\
4 \text { days / week for } \\
6 \text { months }\end{array}$ & $\begin{array}{l}\text { L. paracasei } \\
\text { SD1 }\end{array}$ & $\begin{array}{l}1.8 \times 10^{7} \\
\text { total } \\
\mathrm{CFU} / \mathrm{mL}\end{array}$ & $\begin{array}{l}\text { Significantly lower } \\
\text { counts of MS and } \\
\text { higher levels of } \\
\text { Lactobacilli in saliva } \\
\text { in both probiotic } \\
\text { groups than in the } \\
\text { placebo group. No } \\
\text { difference regarding } \\
\text { these alterations } \\
\text { between the } \\
\text { probiotic groups }\end{array}$ \\
\hline $\begin{array}{l}\text { Lai et al. } \\
\text { (2021) [42] }\end{array}$ & $\begin{array}{c}\text { Randomized, } \\
\text { double-blind, } \\
\text { placebo- } \\
\text { controlled }\end{array}$ & $\begin{array}{l}\text { Children } \\
\text { (4-14 years } \\
\text { old })\end{array}$ & $\begin{array}{l}\text { Type } 1 \\
\text { diabetes } \\
\text { Caries }\end{array}$ & $\begin{array}{c}\text { Test }(n=34) \\
\text { Control }(n=34)\end{array}$ & $\begin{array}{c}2 \text { probiotic } \\
\text { lozenges/day for } \\
60 \text { days } \\
2 \text { placebo } \\
\text { lozenges/day for } \\
60 \text { days }\end{array}$ & $\begin{array}{l}\text { L. brevis } \\
\text { CD2 }\end{array}$ & $\begin{array}{l}2 \times 10^{9} \\
\text { CFU/lozenge }\end{array}$ & $\begin{array}{l}\text { Significant decrease } \\
\text { in salivary MS and in } \\
\text { maximum plaque pH } \\
\text { fall and significant } \\
\text { increase in lowest } \\
\text { plaque pH. }\end{array}$ \\
\hline
\end{tabular}

\section{The Concept of the 'Effector Strain'}

Generally, the 'effector strain' is a microorganism with zero pathogenic potential that can persistently colonize infection-susceptible host tissues and prevent tissues conquest by pathogens. This mechanism has been described as 'replacement therapy'. This method could be used for prevention, as much as for the cure of disease, and potentially could lead to 'herd protection' through effector strain's transmission from one individual to another [101]. In the case of dental caries, the use of certain S. mutans strains as 'effector strains' has been proposed by Hillman [102]. Specific $S$. mutans strains with low acidogenic potential due to a lactate dehydrogenase ( $\mathrm{LDH}$ ) deficiency and the ability to produce particular bacteriocins could be mobilized to serve as 'effector strains' [102-104]. LDH is an enzyme that plays a pivotal role in pyruvate to lactic acid conversion during the catabolism process of glucose by cariogenic bacteria [105]. On the other hand, bacteriocins possess antimicrobial properties against strains or species in close relativity with the producer one [106].

A series of S. mutans strains, JH1000, JH1001, JH1005, JH1010 have been found to produce a specific bacteriocin ('mutacin 1140' or 'MU1140'), a lantibiotic, in particular, with close relativity to nisin's structure [104,107], as the latter has been determined by Hurst [108]. Mutacin 1140 is highly bactericidal against a wide range of microorganisms, primarily Gram-positive (e.g., Streptococcus sanguis, Streptococcus salivarius, Streptococcus pyogenes, Streptococcus mitis, Lactobacillus salivarius, oxacillin-and vancomycin-resistant Staphylococcus aureus and Actinomyces species), and Gram-negative bacteria as well $[104,109,110]$. This is attributed to its ability to powerfully connect with lipid II [111,112], which is a crucial element in bacterial wall synthesis [113] and is targeted by a variety of antibiotics [114]. No adaptive resistance to MU1140 has been presented [109]. JH1000 and its close relatives JH1001, JH1005, and JH1010 were also tested for their ability to colonize rats' oral cavity [104]. JH1001 and JH1005 strains were significantly more competent to displace indigenous S.mutans strains and, conversely, to a lesser extent, displaced by extrinsic bacteria (e.g., S. mutans Ingbritt) than JH1010 strains. The strong correlation between the bacteriocin production and the producer strain's ability to preemptively infect the rodents' oral cavity indicated that JH1001 and its successor, JH1005, could be used as human probiotic strains in the future. The finding reinforced this thesis that JH1001 strain was indeed superinfecting and could displace indigenous $S$. mutans to a great extent in humans, but a minimal infection dose (MID) was not determined [115]. Two years later, 
JH1005's ability to superinfect the human oral cavity was examined [116]. The results were more than encouraging, as $S$. mutans levels significantly decreased 7- and 38-fold in the post-treatment period. In contrast, the rest of the subjects' oral ecological flora remained unaffected, thereby satisfying an excellent precondition for a successful replacement therapy that in no way should effector strains unsettle human oral ecological balance to the extent that predisposition to other diseases is possible.

The key to achieving a fabricated probiotic combining low acidogenicity and high colonizing capacity was introducing the mutant LDH gene into JH1000 strains [117]. Initially, a natural selection favored the wild-type S.mutans JH1000 occurred by eliminating the mutant gene, suggesting that the LDH gene's mutation was lethal in JH1000 [117,118]. This problem could be overcome by limiting the glucose supply and by augmenting alcohol dehydrogenase (ADH) activity, which, as found, compensates for LDH deficiency in high sugar concentrations [119].

With the advent of the new millennium, Hillman et al. announced the construction of an effector strain, BCS3-L1, thanks to the insertion of the adh B gene of ADH (derived from Zymomonas mobilis) to the JH1140 strain (a mutant strain that produces two- to threefold mutacin 1140 than JH1001) [120]. Animal studies highlighted that strain as ideal for replacement therapy inception, as it fulfilled all the prerequisites for an effector strain, which are the significantly reduced pathogenic potential, the selectivity in colonizing the tissues at risk of disease (i.e., the S.mutans niche), genetic stability, superinfecting competency, and prevention of pathogen outgrowth. Targeted mutations were introduced to BCS3-L1 through DNA recombination [50]. These mutations affected the genes dal and come. The dal participates in formation of the bacterial cell wall and the comE gene has a regulatory role in the uptake of exogenous DNA. To date, tests in rats foresee no harmful side effects of A2JM. Collectively, the A2JM strain has low acidogenicity, can colonize the oral cavity, produces high levels of MU1140, and is genetically stable.

Other strains are also under investigation. These include the LDH-deficient S. rattus JH145, which can displace $S$. mutans from the oral cavity of rats [121] and is included in the commercial ProBiora3 TM (Streptococcus uberis KJ2, Streptococcus oralis KJ3, Streptococcus rattus JH145) mouthwash, which is considered a safe and effective adjunct in maintaining dental health [51,122]. A new strategy for replacing dental caries places LDH- and gcrR-deficient and S. mutans in the foreground [123]. Hence, the deletion of this gene allows the LDHdeficient $S$. mutans to better adhere to tooth surfaces. This hypothesis has been confirmed both in vitro and in vivo [124].

At first, the concept of the effector strain constituted a radical notion in probiotic therapy. Now, it is considered an integral part of the contemporary caries cure or prevention strategy in adults. Moreover, the thorough study of the effector strain's capacities and its safety guarantees its clinical effectiveness.

\section{Synbiotics: A New Perspective in Caries Management}

As previously mentioned, the term 'synbiotic' regards products that consist of both probiotics and prebiotics. Gibson and Roberfroid first proposed prebiotics in 1995 to promote symbiosis in gut microbiota [19]. Today, there is clear evidence that prebiotics enhances host's immune function [125-128], selectively favoring health-promoting bacteria, such as Lactobacilli and Bifidobacteria [129-133], employing potential adhesion sites of pathogenic strains, thereby exerting anti-adhesive properties and repressing the virulence of human pathogens per se [130,132,134].

In dentistry, as in general medicine and the food industry [93], prebiotics are combined with probiotics to enhance the latter's ability to "outgrow" pathogens. Until today, only five combinations have been tested; all of them are at pre-clinical stages [135-139]. Glucomannan hydrolysates (GMH) or 3\% galactooligosaccharides (GOS) and 1\% fructooligosaccharides (FOS), when combined with L. acidophilus, suppress S. mutans growth [135-138]. In 2015, Kojima et al. proposed specific probiotic and prebiotic candidates that could be combined to serve as synbiotics [136]. The corresponding probiotics were specific strains 
from the species L. fermentum, L. plantarum, and L. paracasei. The potential prebiotics were xylose, xylitol, and arabinose, which were the only saccharides tested to simultaneously inhibit S. mutans growth and promote the survival of Lactobacilli.

Another strategy of synbiotics evolving in the past few years is incorporating probioticsspecific prebiotics known for their ability to maintain the oral environment's $\mathrm{pH}$ at high levels when a cariogenic challenge occurs. These prebiotics are mainly urea and arginine [140]. Although urea benefits specific oral microorganisms [141-143], its anti-cariogenic effect is notable [144,145], no study about its potential use in synbiotics has been conducted. Arginine, an amino acid strongly positively correlated with caries absence in adults [146], is also well-documented [147]. In contrast to urea, arginine has found one application in synbiotics. This was accomplished by Bijle and his partners [139]. According to this study, arginine concentration is directly correlated with LGG's viability, inhibition of S. mutans per se, biofilm, in general, and lactic acid production, thereby preserving plaque $\mathrm{pH}$ after the treatment application. Nonetheless, clinical trials must be carried out soon to verify their application in the complex oral environment.

Undoubtedly, the concept of synbiotics in caries management has high promise. For the moment, it is still in its infancy. Further clinical trials, which will investigate the in vivo effect of these formulations on the oral microenvironment, especially on dental plaque and its $\mathrm{pH}$, are necessary to clarify whether synbiotics can facilitate our attempts to decrease caries incidence.

\section{Patient Coaching Approach on the Use of Probiotics for Caries Prevention}

Research around probiotics indicates that these may be a good tool in healthcare delivery. It is expected that they will be a cooperative agreement to the patient's adjunct in oral health promotion, as probiotics are simple to consume and do not require any effort from the patients. The latter's compliance to the treatment strongly depends on their attitude towards it and how the dentist could show the short and long-term benefits of following diet instructions. Inevitably, we should persuade dental patients that probiotics are meaningful. This will not be achieved through traditional standardized health advice. Current healthcare advances dictate that the patient should be put in the epicenter and gain an active role in the doctor-patient relationship $[148,149]$. The motivation provided should be based on patients' customized needs and skills. A thorough understanding of the necessity of probiotics in the daily diet and the easy way of consumption they possess in contrast to more demanding oral hygiene practices will ensure their position in all dental patients' diet.

Many alterations are observed from infancy to adolescence as far as oral microbiota and dietary habits are concerned. While the microbiota of children's mouths are unstable, during puberty, they become consistent [100]. Infants and toddlers may be bottle-fed, whereas children and teenagers likely consume high-sugar- or high-starch-containing snacks and beverages $[150,151]$. Both habits favor caries establishment. In the case of infants, toddlers, and children, parents should be informed about the microbiological 'open window'. In this context, milk would be the probiotic carrier of choice, given the fact that they daily consume milk at breakfast time. Teenagers and children also, considering that they may not manage to refrain from a high-sugar diet, should be suggested to prefer probiotic sweetened foods instead of regular ones, as the former are considered to confer health benefits on the consumers $[40,44,55]$.

Adults also have unique dietary patterns which need to be considered [152]. Those between the ages of 18 to 30 years old exhibit no specific nutritional pattern associated with caries disease. Those older than 30 years of age seem to consume high portions of sweetened beverages, sandwiches, and bread, indicating caries prevalence and severity in this group. In high caries-risk adults, the application of the effector strain may be unavoidable.

Older adults often confront serious health and socio-psychological problems, such as obesity, malnutrition, memory lapses, low mood, reduced resilience, etc. [153,154]. Their 
oral health is also compromised [154]. Due to these problems and their advanced age, they subconsciously resist changing their attitude and quit caring about themselves, thereby facing a vicious circle of constant health impairment. This obstacle could be overcome if the dentist learns to encourage such patients and maximize the potential of collaboration and, by extension, of treatment. Considering that probiotics' daily intake does not require excellent skills or daily lives, they may consist of a minor, high profitable diet change for older patients. Again, in these people, the effector strain may have to be chosen for utilization.

People suffering from hyposalivation are vulnerable to oral diseases, including dental caries [155]. Diagnosed hyposalivation often comprises a sequela of severe systemic diseases, such as diabetes mellitus, Sjögren's syndrome, or cancer during the phase of chemotherapy and radiotherapy or may be derived by age or certain drugs [156-162]. In this context, these patients need to be diet coached to admit probiotics into their daily routine. Probiotic lozenges in the daily diet may be the best choice, as lozenges confirmedly increase salivary flow [163]. The inclusion of probiotics will help them surpass the potential jeopardy of caries development or other oral infections. Notably, cancer patients are expected to strictly follow probiotic treatment because they are more receptive to new therapies [164]. During anticancer therapy, the instability of their physical body should need diet highlights to surpass the treatment's stress. Nevertheless, cancer patients are surprisingly unwilling to follow diet recommendations for long; thus, probiotics could be an effective, cheap, and easy solution for positively affecting their oral condition at the first stages of treatment and the phase of maintenance [154].

\section{Discussion}

Dental caries is still a significant public health problem across the world. It has a multifactorial etiology. Health inequality influences general and oral health. In the early 1970s, Swedish children had some of the worst caries statistics in Europe. Accordingly, these inequalities were manifest between groups with lower and higher educational levels. Then, the Swedish government developed a national dental insurance system and proposed that all citizens be entitled to dental care on equal terms. At the same time, they organize public dental care, free of charge, for all children and adolescents up to and including 19 years of age. The result of this politics is the detrimental decline of the caries index. A decline in the incidence of dental caries is also observed in countries having established public health programs using fluoride for dental caries prevention, coupled with changing living conditions, healthier lifestyles, and improved self-care practices. Indeed, the use of fluoride is considered a public health benefit.

Diet also possesses a prominent place in caries establishment and prevention. The frequent consumption of sugars and starches in foods and beverages is the primary causative factor of cariogenesis [165-168]. This etiologic relationship can be mirrored through studies investigating dental caries incidence whenever sugar availability changes occurred $[169,170]$. A correlation of the types of sweetened foods with their cariogenicity does not express the precise action of sugars in the oral cavity in real-time, due to the interplay among sugars with the salivary flow and the preventive measures implemented [171]. Since sugars' role in caries development is evident today, it is sensible to orientate toward their intake confinement. This could be achieved either by reducing sucrose intake (or intake frequency) or replacing sucrose in the diet with sugar substitutes, such as sorbitol and xylitol, or by adding to the diet various food factors, including phosphates [172]. It is not clear yet whether these measures are efficient.

Therefore, as we approach the new era of preventive dentistry, and given that fluoride intake is not a nostrum [173], we should seek contemporary methods that are highly efficient, cost-effective, safe, and necessitate the least involvement of our patients. In general, probiotic strains are considered safe, as most of them have been informally consumed in fermented foods and utilized in general medicine for several years [10,174]. Short-term 
and long-term clinical trials around oral probiotics confirm this, as they do not alter the oral microbial ecology in a direction prone to disease [22,27,28,45].

The effectiveness of the permanence of probiotics' impact on the mouth's microbial flora-and by extension, on oral health-strongly depends on the pre-existent microbial conditions prevailing in the oral cavity [99]. Adults seem not to be favored as much as children at early ages by using probiotics, primarily due to their oral microbiome's stability. This is why we need to consider probiotics better as a preventive method rather than as a therapy per se, meaning that we can implant certain microbial strains in our patients' mouth from an early age to augment the potential benefit to people's oral health in the long-run. Yet, this is not always the case. As it is known, public health measures and pharmaceutical regimens have played a vital role in overall in the dental health improvement and the prolongation of life [175-178]. This, in conjunction with falling birth rates, leads to an aging population $[175,179]$. Given that more people are getting older and preserving their natural teeth than in the past and are more likely to develop caries lesions [180-182], we need to develop methods that are addressing such a population [183]. In these people, the effector strain concept seems to be critical, as it allows us to displace established pathogens at an advanced age, at which naturally significant alterations in oral microecology have not been feasible previously. Perhaps another adult-oriented method to prevent caries lesion in the future may be the autonomous use of mutacin 1140 (MU1140) or its analogues, to confine S. mutans carriage, followed by the treatment of simple probiotic strains, such as L. reuteri strains, to ensure that a desirable integration of theirs in the mouth is competent and durable. The last suggestion is since mutacin 1140 is highly bactericidal for S. mutans [104], this is an ability that can be technically improved [184]. Also, MU1140 is not connected with acquired resistance by pathogens [109], its pharmaco-kinetic and -dynamic properties are well-known $[110,185,186]$, and its analogues can now be produced through laboratory biochemical processes [187].

The most significant advantage of probiotics is that they confer benefits to patients' health through a minimal involvement of the latter. This will make them more acceptable as a new method. Furthermore, probiotics can now be contained in formulations, such as ice-creams and cakes $[40,44,55]$, which contain high proportions of sugars, thereby promoting cariogenesis [171], and make those easily consumed, happy diet delicacies to work in favor of the good oral condition and not against it. The choice to entirely refrain from such products is not feasible, especially for children, who frequently consume a sugar-enriched diet [188]. Thus, the inclusion of probiotic strains in these products would be positive for preventing dental caries as they reduce $S$. mutans salivary levels. This will change in the future; the ways in which diet can benefit oral health, diminishing dental caries, through comfort in use and well-known products, will be accepted by everyone. It seems imperative that different probiotic formulas must be designed in the food industry in collaboration with dental professionals to make oral prevention and human sustainability a fact for future generations.

\section{Conclusions}

The introduction of probiotics to the field of cariology is auspicious for the decrease of caries prevalence. The most important fact of all is that they are addressing a broad spectrum of our patients' ages and health status through multiple manners and they can be readily and safely incorporated into daily use with the application of general coaching models, without necessitating particular toil from the side of the patients. The latter is of great significance for older people. The knowledge around oral probiotics' mechanisms of action, nonetheless, is still lacking. Further studies need to be conducted to understand their interaction with the host's cells and microbiome. In the meantime, they should be used as a preventive method rather than as a caries therapy per se tool. 
Author Contributions: M.A. (Markos Amargianitakis); writing-original draft preparation, M.A. (Maria Antoniadou); writing-review and editing, C.R.; supervision, writing-review and editing, T.V.; project administration, writing-review and editing. All authors have read and agreed to the published version of the manuscript.

Funding: This research received no external funding.

Institutional Review Board Statement: Not applicable.

Informed Consent Statement: Not applicable.

Conflicts of Interest: The authors declare no conflict of interest.

\section{References}

1. Conrads, G.; About, I. Pathophysiology of Dental Caries. Monogr. Oral Sci. 2018, 27, 1-10. [CrossRef]

2. Luan, W.; Baelum, V.; Fejerskov, O.; Chen, X. Ten-Year Incidence of Dental Caries in Adult and Elderly Chinese. Caries Res. 2000, 34, 205-213. [CrossRef] [PubMed]

3. Beikler, T.; Flemmig, T.F. Oral biofilm-associated diseases: Trends and implications for quality of life, systemic health and expenditures. Periodontology 2000 2011, 55, 87-103. [CrossRef]

4. De Amorim, R.G.; Figueiredo, M.J.; Leal, S.C.; Mulder, J.; Frencken, J.E. Caries experience in a child population in a deprived area of Brazil, using ICDAS II. Clin. Oral Investig. 2012, 16, 513-520. [CrossRef] [PubMed]

5. Dye, B.A.; Thornton-Evans, G.; Li, X.; Iafolla, T. Dental Caries and Tooth Loss in Adults in the United States, 2011-2012; NCHS Data Brief, No. 197; US Department of Health and Human Services, Centers for Disease Control and Prevention, National Center for Health Statistics: Hyattsville, MD, USA, 2015.

6. Sweeney, L.C.; Dave, J.; Chambers, P.A.; Heritage, J. Antibiotic resistance in general dental practice-A cause for concern? J. Antimicrob. Chemother. 2004, 53, 567-576. [CrossRef] [PubMed]

7. Qiu, W.; Zhou, Y.; Li, Z.; Huang, T.; Xiao, Y.; Cheng, L.; Peng, X.; Zhang, L.; Ren, B. Application of Antibiotics/ Antimicrobial Agents on Dental Caries. Biomed. Res. Int. 2020, 2020, 5658212. [CrossRef]

8. Teughels, W.; Van Essche, M.; Sliepen, I.; Quirynen, M. Probiotics and oral healthcare. Periodontology 2000 2008, 48, 111-147. [CrossRef]

9. Caglar, E.; Kargul, B.; Tanboga, I. Bacteriotherapy and probiotics' role on oral health. Oral Dis. 2005, 11, 131-137. [CrossRef]

10. Voidarou, C.; Antoniadou, M.; Rozos, G.; Tzora, A.; Skoufos, I.; Varzakas, T.; Lagiou, A.; Bezirtzoglou, E. Fermentative Foods: Microbiology, Biochemistry, Potential Human Health Benefits and Public Health Issues. Foods 2021, 10, 69. [CrossRef]

11. Metchnikoff, I. Lactic acid as inhibiting intestinal putrefactions. In The Prolongation of Life: Optimistic Studies; Metchnikoff, I., Mitchel, P.C., Eds.; Heinemann: London, UK, 1907; pp. 161-183. ISBN 978-0-5989-8207-0.

12. Lilly, D.M.; Stillwell, R.H. Probiotics: Growth-promoting factors produced by microorganisms. Science 1965, 147, 747-748. [CrossRef] [PubMed]

13. Fuller, R. Probiotics in man and animals. J. Appl. Bacteriol. 1989, 66, 365-378.

14. Havenaar, R.; Huis In't Veld, J.H.J. Probiotics: A general view. In The Lactic Acid Bacteria in Health and Disease, 1st ed.; Wood, B.J.B., Ed.; Springer: Boston, MA, USA, 1992; Volume 1, pp. 151-170. ISBN 978-185-166-720-8.

15. Naidu, A.S.; Bidlack, W.R.; Clemens, R.A. Probiotic spectra of lactic acid bacteria (LAB). Crit. Rev. Food Sci. Nutr. 1999, 39, 13-126. [CrossRef] [PubMed]

16. Schrezenmeir, J.; de Vrese, M. Probiotics, prebiotics, and synbiotics-Approaching a definition. Am. J. Clin. Nutr. 2001, 73, 361S-364S. [CrossRef] [PubMed]

17. FAO; WHO. Guidelines for the Evaluation of Probiotics in Food. In Report of a Joint FAO/WHO Working Group on Drafting Guidelines for the Evaluation of Probiotics in Food; FAO/WHO: London, ON, Canada, 2002; Available online: http:/ / www.who.int/ foodsafety/fs_management/en/probiotic_guidelines.pdf (accessed on 15 December 2020).

18. Roberfroid, M. Prebiotics: The Concept Revisited. J. Nutr. 2007, 137, 830S-837S. [CrossRef] [PubMed]

19. Gibson, G.R.; Roberfroid, M.B. Dietary Modulation of the Human Colonic Microbioata: Introducing the Concept of Prebiotics. J. Nutr. 1995, 125, 1401-1412. [CrossRef]

20. Meurman, J.H.; Antila, H.; Salminen, S. Recovery of Lactobacillus Strain GG (ATCC 53103) from Saliva of Healthy Volunteers after Consumption of Yoghurt Prepared with the Bacterium. Microb. Ecol. Health Dis. 1994, 7, 295-298. [CrossRef]

21. Näse, L.; Hatakka, K.; Savilahti, E.; Saxelin, M.; Pönkä, A.; Poussa, T.; Korpela, R.; Meurman, J.H. Effect of Long-Term Consumption of a Probiotic Bacterium, Lactobacillus rhamnosus GG, in Milk on Dental Caries and Caries Risk in children. Caries Res. 2001, 35, 412-420. [CrossRef]

22. Ahola, A.J.; Yli-Knuuttila, H.; Suomalainen, T.; Poussa, T.; Ahlström, A.; Meurman, J.H.; Korpela, R. Short-term consumption of probiotic-containing cheese and its effect on dental caries risk factors. Arch. Oral Biol. 2002, 47, 799-804. [CrossRef]

23. Aminabadi, N.A.; Erfanparast, L.; Ebrahimi, A.; Okouei, S.G. Effect of Chlorhexidine Pretreatment on the Stability of Salivary Latobacilli Probiotic in Six- to Twelve-Year-Old Children: A randomized Controlled Trial. Caries Res. 2011, 45, 148-154. [CrossRef]

24. Juneja, A.; Kakade, A. Evaluating the Effect of Probiotic Containig Milk on Salivary mutans streptococci Levels. J. Clin. Pediatr. Dent. 2012, 37, 9-14. [CrossRef] 
25. Stecksen-Blicks, C.; Sjöström, I.; Twetman, S. Effect of Long-Term Consumption of Milk Supplemented with Probiotic Lactobacilli and Fluoride on Dental Caries and General Health in Preschool Children: A Cluster Randomized Study. Caries Res. 2009, 43, 374-381. [CrossRef] [PubMed]

26. Nikawa, H.; Makihira, S.; Fukushima, H.; Nishimura, H.; Ozaki, Y.; Ishida, K.; Darmawan, S.; Hamada, T.; Hara, K.; Matsumoto, A.; et al. Lactobacillus reuteri in bovine milk fermented decreases the oral carriage of mutans streptococci. Int. J. Food Microbiol. 2004, 95, 219-223. [CrossRef] [PubMed]

27. Caglar, E.; Kavaloglu-Cildir, S.; Ergeneli, S.; Sandalli, N.; Twetman, S. Salivary mutans streptococci and lactobacilli levels after ingestion of the probiotic bacterium Lactobacillus reuteri ATCC 55730 by straws or tablets. Acta Odontol. Scand. 2006, 64, 314-318. [CrossRef]

28. Stensson, M.; Koch, G.; Coric, S.; Abrahamsson, T.R.; Jenmalm, M.C.; Birkhed, D.; Wendt, L.K. Oral Administration of Lactobacillus reuteri during the First Year of Life Reduces Caries Prevalence in the Primary Dentition at 9 Years of Age. Caries Res. 2014, 48, 111-117. [CrossRef]

29. Kavaloglu-Cildir, S.; Sandalli, N.; Nazli, S.; Alp, F.; Caglar, E. A Novel Delivery System of Probiotic Drop and its Effect on Dental Caries Risk Factors in Cleft Lip/Palate Children. Cleft Palate Craniofac. J. 2012, 49, 369-372. [CrossRef]

30. Keller, M.K.; Nohr Larsen, I.; Karlsson, I.; Twetman, S. Effect of tablets containing probiotic bacteria (Lactobacillus reuteri) on early caries lesions in adolescents: A pilot study. Benef. Microbes 2014, 5, 403-407. [CrossRef]

31. Gizani, S.; Petsi, G.; Twetman, S.; Caroni, C.; Makou, M.; Papagianoulis, L. Effect of the probiotic bacterium Lactobacillus reuteri on white spote lesion development in orthodontic patients. Eur. J. Orthod. 2016, 38, 85-89. [CrossRef]

32. Alamoudi, N.M.; Almabadi, E.S.; El Ashiry, E.A.; El Derwi, D.A. Effect of Probiotic Lactobacillus reuteri on salivary Cariogenic Bacterial Counts among Groups of Preschool Children in Jeddah, Saudi Arabia: A randomized Clinical Trial. J. Clin. Pediatr. Dent. 2018, 42, 331-338. [CrossRef]

33. Yadav, M.; Poornima, P.; Roshan, N.M.; Prachi, N.; Veena, M.; Neena, I.E. Evaluation of Probiotic Milk on Salivary Mutans Streptococci Count: An In Vivo Microbiological Study. J. Clin. Pediatr. Dent. 2014, 39, 23-26. [CrossRef]

34. Hasslöf, P.; West, C.E.; Karlsson Videhult, F.; Brandelius, C.; Stecksen-Blicks, C. Early Intervention with Probiotic Lactobacillus paracasei F19 Has No Long-Term Effect on Caries Experience. Caries Res. 2013, 47, 559-565. [CrossRef] [PubMed]

35. Chuang, L.C.; Huang, C.S.; Ou-Yang, L.W.; Lin, S.Y. Probiotic Lactobacillus paracasei effect on cariogenic bacterial flora. Clin. Oral Investig. 2011, 15, 471-476. [CrossRef]

36. Teanpaisan, R.; Piwat, S. Lactobacillus paracasei SD1, a novel probiotic, reduces mutans streptococci in human volunteers: A randomized placebo-controlled trial. Clin. Oral Investig. 2014, 18, 857-862. [CrossRef]

37. Pahumunto, N.; Piwat, S.; Chankanka, O.; Akkarachaneeyakorn, N.; Rangsitsathian, K.; Teanpaisan, R. Reducing mutans streptococci and caries development by Lactobacillus paracasei SD1 in preschool children: A randomized placebo-controlled trial. Acta Odontol. Scand. 2018, 76, 331-337. [CrossRef] [PubMed]

38. Manmontri, C.; Nirunsittirat, A.; Piwat, S.; Wattanarat, O.; Pahumunto, N.; Makeudom, A.; Sastraruji, T.; Krisanaprakornkit, S.; Teanpaisan, R. Reduction of Streptococcus mutans by probiotic milk: A multicenter randomized controlled trial. Clin. Oral Investig. 2020, 24, 2363-2374. [CrossRef] [PubMed]

39. Ghasemi, E.; Mazaheri, R.; Tahmourespour, A. Effect of Probiotic Yogurt and Xylitol-Containing Chewing Gums on Salivary $S$ Mutans Count. J. Clin. Pediatr. Dent. 2017, 41, 257-263. [CrossRef] [PubMed]

40. Singh, R.P.; Damle, S.G.; Chawla, A. Salivary mutans streptococci and lactobacilli modulations in young children on consumption of probiotic ice-cream containing Bifidobacterium lactis Bb-12 and Lactobacillus La5. Acta Odontol. Scand. 2011, 69, 389-394. [CrossRef]

41. Nishihara, T.; Suzuki, N.; Yoneda, M.; Hirofuji, T. Effects of Lactobacillus salivarius-containing tablets on caries risk factors: A randomized open-label clinical trial. BMC Oral Health 2014, 14, 110. [CrossRef]

42. Lai, S.; Lingström, P.; Cagetti, M.G.; Cocco, F.; Meloni, G.; Arrica, M.A.; Campus, G. Effect of Lactobacillus brevis CD2 containing lozenges and plaque $\mathrm{pH}$ and cariogenic bacteria in diabetic children: A randomised clinical trial. Clin. Oral Investig. 2021, 25, 115-123. [CrossRef]

43. Montalto, M.; Vastola, M.; Marigo, L.; Covino, M.; Graziosetto, R.; Curigliano, V.; Santoro, L.; Cuoco, L.; Manna, R.; Gasbarrini, G. Probiotic Treatment Increases Salivary Counts of Lactobacilli: A Double-Blind, Randomized, Controlled Study. Digestion 2004, 69, 53-56. [CrossRef]

44. Caglar, E.; Kuscu, O.O.; Selvi Kuvvetli, S.; Kavaloglu Cildir, S.; Sandalli, N.; Twetman, S. Short-term effect of ice-cream containing Bifidobacterium lactis Bb-12 on the number of salivary mutans streptococci and lactobacilli. Acta Odontol Scand 2008, 66, 154-158. [CrossRef]

45. Taipale, T.; Pienihäkkinen, K.; Salminen, S.; Jokela, J.; Söderling, E. Bifidobacterium animalis subsp. Lactis BB-12 Administration in Early Childhood: A randomized Clinical Trial of Effects on Oral Colonization by Mutans Streptococci and the Probiotic. Caries Res. 2012, 46, 69-77. [CrossRef]

46. Javid, A.Z.; Amerian, E.; Basir, L.; Ekrami, A.; Haghighizadeh, M.H.; Maghsoumi-Norouzabad, L. Effects of the Consumption of Probiotic Yogurt Containing Bifidobacterium lactis Bb12 on the Levels of Streptococcus mutans and Lactobacilli in Saliva of Students with Initial Stages of Dental Caries: A Double-Blind Randomized Controlled Trial. Caries Res. 2020, 54, 68-74. [CrossRef]

47. Caglar, E.; Sandalli, N.; Twetman, S.; Kavaloglu, S.; Ergeneli, S.; Selvi, S. Effect of yogurt with Bifidobacterium DN-173 010 on salivary mutans streptococci and lactobacilli in young adults. Acta Odontol. Scand. 2005, 63, 317-320. [CrossRef] 
48. Pinto, G.S.; Cenci, M.S.; Azevedo, M.S.; Epifanio, M.; Jones, M.H. Effect of Yogurt Containing Bifidobacterium animalis subsp. lactis DN-173010 Probiotic on Dental Plaque and Saliva in Orthodontic Patients. Caries Res. 2014, 48, 63-68. [CrossRef]

49. Villavicencio, J.; Villegas, L.M.; Arango, M.C.; Arias, S.; Triana, F. Effects of a food enriched with probiotics on Streptococcus mutans and Lactobacillus spp. salivary counts in preschool children: A cluster randomized trial. J. Appl. Oral Sci. 2018, 26, e20170318. [CrossRef]

50. Hillman, J.D.; Mo, J.; McDonell, E.; Cvitkovitch, D.; Hillman, C.H. Modification of an effector strain for replacement therapy of dental caries to enable clinical safety trials. J. Appl. Microbiol. 2007, 102, 1209-1219. [CrossRef]

51. Zahradnik, R.T.; Magnusson, I.; Walker, C.; McDonell, E.; Hillman, C.H.; Hillman, J.D. Preliminary assessment of safety and effectiveness in humans of ProBiora3 TM, a probiotic mouthwash. J. Appl. Microbiol. 2009, 107, 682-690. [CrossRef] [PubMed]

52. Hedayati-Hajikand, T.; Lundberg, U.; Eldh, C.; Twetman, S. Effect of probiotic chewing tablets on early childhood caries-A randomized controlled trial. BMC Oral Health 2015, 15. [CrossRef]

53. Ferrer, M.D.; Lopez-Lopez, A.; Nicolescu, T.; Salavert, A.; Mendez, I.; Cune, J.; Llena, C.; Mira, A. A pilot study to assess oral colonization and $\mathrm{pH}$ buffering by the probiotic Streptococcus dentisani under different dosing regimes. Odontology 2020, 108, 180-187. [CrossRef] [PubMed]

54. Burton, J.P.; Drummond, B.K.; Chilcott, C.N.; Tag, J.R.; Thomson, W.M.; Hale, J.D.F.; Wescombe, P.A. Influence of the probiotic Streptococcus salivarius strain M18 on indices of dental health in children: A randomized double-blind, placebo-controlled trial. J. Med. Microbiol. 2013, 62, 875-884. [CrossRef] [PubMed]

55. Koopaie, M.; Fatahzadeh, M.; Jahangir, S.; Bakhtiari, R. Comparison of the effect of regular and probiotic cake (Bacillus coagulans) on salivary $\mathrm{pH}$ and Streptococcus mutans count. Dent. Med. Probl. 2019, 56, 33-38. [CrossRef] [PubMed]

56. Teughels, W.; Loozen, G.; Quirynen, M. Do probiotics offer opportunities to manipulate the periodontal oral microbiota? J. Clin. Periodontol. 2011, 38, 159-177. [CrossRef] [PubMed]

57. Delcenserie, V.; Martel, D.; Lamoureux, M.; Amiot, K.; Boutin, Y.; Roy, D. Immunomodulatory Effects of Probiotics in the Intestinal Tract. Curr. Iss. Mol. Biol. 2008, 10, 37-54.

58. Schiffrin, E.J.; Brassart, D.; Selvin, A.L.; Rochat, F.; Donnet-Hughes, A. Immune modulation of blood leukocytes in humans by lactic acid bacteria: Criteria for strain selection. Am. J. Clin. Nutr. 1997, 66, 515S-520S. [CrossRef]

59. Pelto, L.; Isolauri, E.; Lilius, E.M.; Nuutila, J.; Salminen, S. Probiotic bacteria down-regulate the milk-induced inflammatory response in milk-hypersensitive subjects but have an immunostimulatory effect in healthy subjects. Clin. Exp. Allergy 1998, 28, 1474-1479. [CrossRef]

60. Schiffrin, E.J.; Rochat, F.; Link-Amster, H.; Aeschlimann, J.M.; Donnet-Hughes, A. Immunomodulation of human blood cells following the ingestion of lactic acid bacteria. J. Dairy Sci. 1995, 78, 491-497. [CrossRef]

61. Donnet-Hughes, A.; Rochat, F.; Serrant, P.; Aeschlimann, J.M.; Schiffrin, E.J. Modulaion of nonspecific mechanisms of defense by lactic acid bacteria: Effective dose. J. Dairy Sci. 1999, 82, 863-869. [CrossRef]

62. Kaila, M.; Isolauri, E.; Saxelin, M.; Arvilommi, H.; Vesikari, T. Viable versus inactivated Lactobacillus strain GG in acute rotavirus diarrhea. Arch. Dis. Child. 1995, 72, 51-53. [CrossRef]

63. Majamaa, H.; Isolauri, E.; Saxelin, M.; Vesikari, T. Lactic acid bacteria in the treatment of acute rotavirus gastroenteritis. J. Pediatr. Gastroenterol. Nutr. 1995, 20, 333-338. [CrossRef]

64. Fukushima, Y.; Kawata, Y.; Hara, H.; Terada, A.; Mitsuoka, T. Effect of a probiotic formula on intestinal immunoglobulin A production in healthy children. Int. J. Food. Microbiol. 1998, 42, 39-44. [CrossRef]

65. Cosseau, C.; Devine, D.A.; Dullaghan, E.; Gardy, J.L.; Chikatamarla, A.; Gellatly, S.; Yu, L.L.; Pistolic, J.; Falsafi, R.; Tagg, J.; et al. The commensal Streptococcus salivarius K12 downregulates the innate immune responses of human epithelial cells and promotes host-microbe homeostasis. Infect. Immun. 2008, 76, 4163-4175. [CrossRef]

66. Zhang, G.; Chen, R.; Rudney, J.D. Streptococcus cristatus attenuates Fusobacterium nucleatum-induced interleukin-8 expression in oral epithelial cells. J. Periodontal. Res. 2008, 43, 408-416. [CrossRef] [PubMed]

67. Sliepen, I.; Van Damme, J.; Van Essche, M.; Loozen, G.; Quirynen, M.; Teughels, W. Microbial interactions influence inflammatory host cell responses. J. Dent. Res. 2009, 88, 1026-1030. [CrossRef] [PubMed]

68. Della Riccia, D.N.; Bizzini, F.; Perilli, M.G.; Polimeni, A.; Trinchieri, V.; Amicosante, G.; Cifone, M.G. Anti-inflammatory effects of Lactobacillus brevis (CD2) on periodontal disease. Oral Dis. 2007, 13, 376-385. [CrossRef] [PubMed]

69. Ganz, T. Defensins: Antimicrobial peptides of innate immunity. Nat. Rev. Immunol. 2003, 3, 710-720. [CrossRef]

70. Wattanarat, O.; Makeudom, A.; Sastraruji, T.; Piwat, S.; Tianviwat, S.; Teanpaisan, R.; Krisanaprakornkit, S. Enhancement of salivary human neutrophil peptide 1-3 levels by probiotic supplementation. BMC Oral Health 2015, 15, 19. [CrossRef] [PubMed]

71. Gillor, O.; Etzion, A.; Riley, M.A. The dual role of bacteriocins as anti- and probiotics. Appl. Microbiol. Biotechnol. 2008, 81, 591-606. [CrossRef]

72. Gordon, D.M. The potential of bacteriocin-producing probiotics and associated caveats. Future Microbiol. 2009, 4, 941-943. [CrossRef] [PubMed]

73. Oelschlaeger, T.A. Mechanisms of probiotic actions-A review. Int. J. Med. Microbiol. 2010, 300, 57-62. [CrossRef] [PubMed]

74. Silva, M.; Jacobus, N.V.; Deneke, C.; Gorbach, S.L. Antimicrobial Substance from a human Lactobacillus Strain. Antimicrob. Agents Chemother. 1987, 31, 1231-1233. [CrossRef]

75. Axelsson, L.T.; Chung, T.C.; Dobrogosz, W.J.; Lindgren, S.E. Production of a Broad Spectrum Antimicrobial Substance by Lactobacillus reuteri. Microb. Ecol. Health Dis. 1989, 2, 131-136. [CrossRef] 
76. Gänzle, M.G.; Höltzel, A.; Walter, J.; Jung, G.; Hammes, W.P. Characterization of Reutericyclin Produced by Lactobacillus reuteri LTH2584. Appl. Environ. Microbiol. 2000, 66, 4325-4333. [CrossRef] [PubMed]

77. Schaefer, L.; Auchtung, T.A.; Hermans, K.E.; Whitehead, D.; Borhan, B.; Britton, R.A. The antimicrobial compound reuterin (3-hydroxypropionaldehyde) induces oxidative stress via interaction with thiol groups. Microbiology 2010, 156, 1589-1599. [CrossRef] [PubMed]

78. Gänzle, M.G.; Vogel, R.F. Studies on the Mode of Action of Reutericyclin. Appl. Environ. Microbiol. 2003, 69, 1305-1307. [CrossRef] [PubMed]

79. Elli, M.; Zink, R.; Rytz, A.; Reniero, R.; Morelli, L. Iron requirement of Lactobacillus spp. in completely chemically defined growth media. J. Appl. Microbiol. 2000, 88, 695-703. [CrossRef]

80. Teughels, W.; Kinder Haake, S.; Sliepen, I.; Pauwels, M.; Van Eldere, J.; Cassiman, J.J.; Quirynen, M. Bacteria interfere with A. actinomycetemcomitans colonization. J. Dent. Res. 2007, 86, 611-617. [CrossRef]

81. Sliepen, I.; Hofkens, J.; Van Essche, M.; Quirynen, M.; Teughels, W. Aggregatibacter actinomycetemcomitans adhesion inhibited in a flow cell. Oral Microbiol. Immunol. 2008, 23, 520-524. [CrossRef]

82. Van Hoogmoed, C.G.; van der Kuijl-Booij, M.; van der Mei, H.C.; Busscher, H.J. Inhibition of Streptococcus mutans NS Adhesion to Glass with and without a Salivary Conditioning Film by Biosurfactant-Releasing Streptococcus mitis Strains. Appl. Environ. Microbiol. 2000, 66, 659-663. [CrossRef]

83. Haukioja, A.; Loimaranta, V.; Tenovuo, J. Probiotic bacteria affect the composition of salivary pellicle and Streptococcal adhesion in vitro. Oral Microbiol. Immunol. 2008, 23, 336-343. [CrossRef] [PubMed]

84. Marsh, P.D.; Martin, M.V. Plaque-mediated diseases: Dental caries and periodontal diseases. In Marsh and Martin's Oral Microbiology, 6th ed.; Marsh, P.D., Lewis, M.A.O., Rogers, H., Williams, D.W., Wilson, M., Eds.; Elsevier Ltd.: Amsterdam, Netherlands, 2016; pp. 112-129. ISBN 978-070-206-106-6.

85. Marsh, P.D. Dental Biofilms in Health and Disease. In Understanding Dental Caries: From Pathogenesis to Prevention and Therapy, 1st ed.; Goldberg, M., Ed.; Springer International Publishing: Cham, Switzerland, 2016; pp. 41-52. ISBN 978-3-319-80837-6.

86. Keyes, P.H. Research in dental caries. J. Am. Dent. Assoc. 1968, 76, 1357-1373. [CrossRef]

87. Simon-Soro, A.; Belda-Ferre, P.; Cabrera-Rubio, R.; Alcaraz, L.D.; Mira, A. A Tissue-Dependent Hypothesis of Dental Caries. Caries Res. 2013, 47, 591-600. [CrossRef]

88. Gedalia, I.; Dakuar, A.; Shapira, L.; Lewinstein, I.; Goultschin, J.; Rahamin, E. Enamel softening with Coca Cola and rehardening with milk or saliva. Am. J. Dent. 1991, 4, 120-122.

89. Bowen, W.H.; Pearson, S.K. Effect of milk on cariogenesis. Caries Res. 1993, 27, 461-466. [CrossRef] [PubMed]

90. Kashket, S.; Yaskell, T. Effectiveness of calcium lactate added to food in reducing intraoral demineralization of enamel. Caries Res. 1997, 31, 429-433. [CrossRef] [PubMed]

91. Gorbach, S.L.; Goldin, B.R. Lactobacillus strains and methods of selection. US Patent Application No. 4,839,281, 13 June 1989.

92. Hatakka, K.; Savilahti, E.; Pönkä, A.; Meurman, J.H.; Poussa, T.; Näse, L.; Saxelin, M.; Korpela, R. Effect of long term consumption of probiotic milk on infections in children attending day care centres: Double blind, randomized trial. BMJ 2001, 322, 1327-1329. [CrossRef]

93. Antoniadou, M.; Varzakas, T. Probiotics and Prebiotics and Their Effect on Food and Human Health: New Perspectives. In Probiotics, the Natural Microbiota in Living Organisms: Fundamental and Applications; El Enshasy, H.A., Yang, S.T., Eds.; CRC Press: Boca Raton, FL, USA, 2021; ISBN 978-113-849-360-5.

94. Grudianov, A.I.; Dmitrieva, N.A.; Fomenko, E.V. Use of probiotics Bifidumbacterin and Acilact in tablets in therapy of periodontal inflammations. Stomatol. Mosk. 2002, 81, 39-43.

95. Van Houte, J. Role of Micro-organisms in Caries Etiology. J. Dent. Res. 1994, 73, 672-681. [CrossRef]

96. Busscher, H.J.; Mulder, A.F.J.M.; van der Mei, H.C. In vitro Adhesion to Enamel and in vivo Colonization of Tooth Surfaces by Lactobacilli from a Bio-Yoghurt. Caries Res. 1999, 33, 403-404. [CrossRef]

97. Petti, S.; Tarsitani, G.; D'Arca, A.S. A randomized clinical trial of the effect of yoghurt on the human salivary microflora. Arch. Oral Biol. 2001, 46, 705-712. [CrossRef]

98. Yli-Knuuttila, H.; Snäll, J.; Kari, K.; Meurman, J.H. Colonization of Lactobacillus rhamnosus GG in the oral cavity. Oral Microbiol. Immunol. 2006, 21, 129-131. [CrossRef] [PubMed]

99. Devine, D.A.; Marsh, P.D. Prospects for the development of probiotics and prebiotics for oral applications. J. Oral Microbiol. 2009, 1, 1949. [CrossRef]

100. Killian, M.; Frandsen, E.V.G.; Haubek, D.; Poulsen, K. The etiology of periodontal disease revisited by population genetic analysis. Periodontology 2000 2006, 42, 158-179. [CrossRef]

101. Hillman, J.D.; Socransky, S.S. Replacement therapy for the prevention of dental disease. Adv. Dent. Res. 1987, 1, 119-125. [CrossRef]

102. Hillman, J.D. Lactate Dehydrogenase Mutants of Streptococcus mutans: Isolation and Preliminary Characterization. Infect. Immun. 1978, 21, 206-212. [CrossRef]

103. Ikeda, T.; Iwanami, T.; Hirasawa, M.; Watanabe, C.; McGhee, J.R.; Shiota, T. Purification and Certain Properties of a Bacteriocin from Streptococcus mutans. Infect. Immun. 1982, 35, 861-868. [CrossRef] [PubMed]

104. Hillman, J.D.; Johnson, K.P.; Yaphe, B.I. Isolation of a Streptococcus mutans Strain Producing a Novel Bacteriocin. Infect. Immun. 1984, 44, 141-144. [CrossRef] 
105. Brown, A.T.; Wittenberger, C.L. Fructose-1,6-Diphosphate-Dependent Lactate Dehydrogenase from a Cariogenic Streptococcus: Purification and Regulatory Properties. J. Bacteriol. 1972, 110, 604-615. [CrossRef] [PubMed]

106. Reeves, P. The Bacteriocins. Bacteriol. Rev. 1965, 29, 24-45. [CrossRef]

107. Hillman, J.D.; Novak, J.; Sagura, E.; Gutierrez, J.A.; Brooks, T.A.; Crowley, P.J.; Hess, M.; Azizi, A.; Leung, K.P.; Cvitkovitch, D.; et al. Genetic and Biochemical Analysis of Mutacin 1140, a Lantibiotic from Streptococcus mutans. Infect. Immun. 1998, 66, 2743-2749. [CrossRef] [PubMed]

108. Hurst, A. Nisin. Adv. Appl. Microbiol. 1981, 27, 85-123. [CrossRef]

109. Ghobrial, O.G.; Derendorf, H.; Hillman, J.D. Pharmacodynamic activity of the lantibiotic MU1140. Int. J. Antimicrob. Agents 2009, 33, 70-74. [CrossRef]

110. Ghobrial, O.G.; Derendorf, H.; Hillman, J.D. Pharmacokinetic and Pharmacodynamic Evaluation of the Lantibiotic MU1140. J. Pharm. Sci. 2010, 99, 2521-2528. [CrossRef] [PubMed]

111. Hasper, H.E.; Kramer, N.E.; Smith, J.L.; Hillman, J.D.; Zachariah, C.; Kuipers, O.P.; de Kruijff, B.; Breukink, E. An Alternative Bactericidal Mechanism of Action for Lantibiotic Peptides That Target Lipid II. Science 2006, 313, 1636-1637. [CrossRef]

112. Smith, L.; Hasper, H.; Breukink, E.; Novak, J.; Cerkasov, J.; Hillman, J.D.; Wilson-Stanford, S.; Orugunty, R.S. Elucidation of the Antimicrobial Mechanism of Mutacin 1140. Biochemistry 2008, 47, 3308-3314. [CrossRef] [PubMed]

113. De Kruijff, B.; van Dam, V.; Breukink, E. Lipid II: A central component in bacterial cell wall synthesis and a target for antibiotics. Prostaglandins Leukot. Essent. Fatty Acids 2008, 79, 117-121. [CrossRef]

114. Malin, J.J.; de Leeuw, E. Therapeutic compounds targeting Lipid II for antibacterial purposes. Infect. Drug. Resist. 2019, 12, 2613-2625. [CrossRef] [PubMed]

115. Hillman, J.D.; Yaphe, B.I.; Johnson, K.P. Colonization of the Human Oral Cavity by a Strain of Streptococcus mutans. J. Dent. Res. 1985, 64, 1272-1274. [CrossRef]

116. Hillman, J.D.; Dzuback, A.L.; Andrews, S.W. Colonization of the Human Oral Cavity by a Streptococcus mutans Mutant Producing Increased Bacteriocin. J. Dent. Res. 1987, 66, 1092-1094. [CrossRef]

117. Hillman, J.D.; Chen, A.; Duncan, M.; Lee, S.W. Evidence that L-(+)-Lactate Dehydrogenase Deficiency Is Lethal in Streptococcus mutans. Infect. Immun. 1994, 62, 60-64. [CrossRef] [PubMed]

118. Chen, A.; Hillman, J.D.; Duncan, M. L-(+)-Lactate Dehyrdrogenase Deficiency is Lethal in Streptococcus mutans. J. Bacteriol. 1994, 176, 1542-1545. [CrossRef]

119. Hillman, J.D.; Chen, A.; Snoep, J.L. Genetic and Physiological Analysis of the Lethal Effect of L-(+)-Lactate Dehydrogenase Deficiency in Streptococcus mutans: Complementation by Alcohol Dehydrogenase from Zymomonas mobilis. Infect. Immun. 1996, 64, 4319-4323. [CrossRef]

120. Hillman, J.D.; Brooks, T.A.; Michalek, S.M.; Harmon, C.C.; Snoep, J.L.; van der Weijden, C.C. Construction and Characterization of an Effector Strain of Streptococcus mutans for Replacement Therapy of Dental Caries. Infect. Immun. 2000, 68, 543-549. [CrossRef] [PubMed]

121. Hillman, J.D.; McDonell, E.; Cramm, T.; Hillman, C.H.; Zahradnik, R.T. A spontaneous lactate dehydrogenase deficient mutant of Streptococcus rattus for use as a probiotic in the prevention of dental caries. J. Appl. Microbiol. 2009, 107, 1551-1558. [CrossRef]

122. Hillman, J.D.; McDonell, E.; Hillman, C.H.; Zahradnik, R.T.; Soni, M.G. Safety Assessment of ProBiora3, a Probiotic Mouthwash: Subchronic Toxicity Study in Rats. Int. J. Toxicol. 2009, 28, 357-367. [CrossRef]

123. Sun, J.H.; Xu, Q.A.; Fan, M.W. A new strategy for the replacement therapy of dental caries. Med. Hypotheses 2009, 73, 1063-1064. [CrossRef] [PubMed]

124. Pan, W.; Mao, T.; Xu, Q.A.; Shao, J.; Liu, C.; Fan, M. A New gcrR-Deficient Streptococcus mutans Mutant for Replacement Therapy of Dental Caries. Sci. World J. 2013, 2013, 460202. [CrossRef] [PubMed]

125. Swanson, K.S.; Grieshop, C.M.; Flickinger, E.A.; Bauer, L.L.; Healy, H.P.; Dawson, K.A.; Merchen, N.R.; Fahey, G.C., Jr. Supplemental Fructooligosaccharides and Mannanoligosaccharides Influence Immune Function, Ileal and Total Tract Nutrient Digestibilities, Microbial Populations and Concentrations of Protein Catabolites in the Large Bowel of Dogs. J. Nutr. 2002, 132, 980-989. [CrossRef]

126. Yamada, K.; Tokunaga, Y.; Ikeda, A.; Ohkura, K.; Kaku-Ohkura, S.; Mamiya, S.; Ou Lim, B.; Tachibana, H. Effect of Dietary Fiber on the Lipid Metabolism and Immune Function of Aged Sprague-Dawley Rats. Biosci. Biotechnol. Biochem. 2003, 67, 429-433. [CrossRef]

127. Forchielli, M.L.; Walker, W.A. The role of gut-associated lymphoid tissues and mucosal defence. Br. J. Nutr. 2005, 93, S41-S48. [CrossRef] [PubMed]

128. Torrecillas, S.; Makol, A.; Caballero, M.J.; Montero, D.; Robaina, L.; Real, F.; Sweetman, J.; Tort, L.; Izquierdo, M.S. Immune stimulation and improved infection resistance in European sea bass (Dicentrarchus labrax) fed mannan oligosaccharides. Fish Shellfish Immunol. 2007, 23, 969-981. [CrossRef]

129. Gibson, G.R.; Beatty, E.R.; Wang, X.; Cummings, J.H. Selective Stimulation of Bifidobacteria in the Human Colon by Oligofructose and Inulin. Gastroenterology 1995, 108, 975-982. [CrossRef]

130. Gibson, G.R.; McCartney, A.L.; Rastall, R.A. Prebiotics and resistance to gastrointestinal infections. Br. J. Nutr. 2005, 93, S31-S34. [CrossRef] [PubMed]

131. Kleessen, B.; Blaut, M. Modulation of gut mucosal biofilms. Br. J. Nutr. 2005, 93, S35-S40. [CrossRef] [PubMed] 
132. Al-Ghazzewi, F.H.; Khanna, S.; Tester, R.F.; Piggott, J. The potential use of hydrolysed konjac glucomannan as a prebiotic. J. Sci. Food Agric. 2007, 87, 1758-1766. [CrossRef]

133. Elamir, A.A.; Tester, R.F.; Al-Ghazzewi, F.H.; Kaal, H.Y.; Ghalbon, A.A.; Elmegrahai, N.A.; Piggott, J.R. Effects of konjac glucomannan hydrolysates on the gut microflora of mice. Nutr. Food. Sci. 2008, 38, 422-429. [CrossRef]

134. Park, S.F.; Kroll, R.G. Expression of listeriolysin and phosphatidylinositol specific phospholipace C is repressed by the plantderived molecule cellobiose in Listeria monocytogenes. Mol. Microbiol. 1993, 8, 653-661. [CrossRef] [PubMed]

135. Tester, R.F.; Al-Ghazzewi, F.H. A preliminary study of the synbiotic effects of konjac glucomannan hydrolysates (GMH) and lactobacilli on the growth of oral bacterium Streptococcus mutans. Nutr. Food Sci. 2011, 41, 234-237. [CrossRef]

136. Kojima, Y.; Ohshima, T.; Seneviratne, C.J.; Maeda, N. Combining prebiotics and probiotics to develop novel synbiotics that suppress oral pathogens. J. Oral. Biosci. 2016, 58, 27-32. [CrossRef]

137. Nunpan, S.; Suwannachart, C.; Wayakanon, K. The Inhibition of Dental Caries Pathogen by Using Prebiotic and Probiotic Combination. J. Dent. Assoc. Thai. 2017, 67, 31-38.

138. Nunpan, S.; Suwannachart, C.; Wayakanon, K. Effect of Prebiotics-Enhanced Probiotics on the Growth of Streptococcus mutans. Int. J. Microbiol. 2019, 2019, 4623807. [CrossRef]

139. Bijle, M.N.; Neelakantan, P.; Ekambaram, M.; Lo, E.C.M.; Yiu, C.K.Y. Effect of a novel synbiotic on Streptococcus mutans. Sci. Rep. 2020, 10, 7951. [CrossRef] [PubMed]

140. Burne, R.A.; Marquis, R.E. Alkali production by oral bacteria and protection against dental caries. FEMS Microbiol. Lett. 2000, 193, 1-6. [CrossRef] [PubMed]

141. Chen, Y.Y.; Weaver, C.A.; Burne, R.A. Dual functions of Streptococcus salivarius urease. J Bacteriol 2000, 182, 4667-4669. [CrossRef]

142. Liu, Y.; Hu, T.; Zhang, J.; Zhou, X. Characterization of the Actinomyces naeslundii ureolysis and its role in bacterial aciduricity and capacity to modulate $\mathrm{pH}$ homeostasis. Microbiol. Res. 2006, 161, 304-310. [CrossRef] [PubMed]

143. Morou-Bermundez, E.; Burne, R.A. Analysis of urease expression in Actinomyces naeslundii WVU45. Infect. Immun. 2000, 68, 6670-6676. [CrossRef] [PubMed]

144. Stephan, R.M. The effect of urea in counteracting the influence of carbohydrates on the $\mathrm{pH}$ of dental plaques. J. Dent. Res. 1943, 22, 63-71. [CrossRef]

145. Clancy, K.A.; Pearson, S.; Bowen, W.H.; Burne, R.A. Characterization of recombinant, ureolytic Streptococcus mutans demonstrates an inverse relationship between dental plaque ureolytic capacity and cariogenicity. Infect. Immun. 2000, 68, 2621-2629. [CrossRef] [PubMed]

146. Van Wuyckhuyse, B.C.; Perinpanayagam, H.E.R.; Bevacqua, D.; Raubertas, R.E.; Billings, R.J.; Bowen, W.H.; Tabak, L.A. Association of free arginine and lysin concentrations in human parotid saliva with caries experience. J. Dent. Res. 1995, 74, 686-690. [CrossRef]

147. Nascimento, M.M. Potential Uses of Arginine in Dentistry. Adv. Dent. Res. 2018, 29, 98-103. [CrossRef]

148. Antoniadou, M. Delivering Self-Management Health Outcomes Using the Patient Activation Measure Instrument-Prospects for the Dentistry Field. Adv. Dent. Oral Health 2020, 12, 555833.

149. Antoniadou, M.; Varzakas, T. Health and Oral Health Coaching Issues. Encyclopedia 2020. Available online: https:/ / encyclopedia. pub/1755 (accessed on 1 April 2021).

150. Avila, W.M.; Pordeus, I.A.; Paiva, S.M.; Martins, C.C. Breast and Bottle Feeding as Risk Factors for Dental Caries: A Systematic Review and Meta-Analysis. PLoS ONE 2015, 10, e0142922. [CrossRef]

151. Reddy, A.; Norris, D.F.; Momeni, S.S.; Waldo, B.; Ruby, J.D. The pH of beverages in the United States. J. Am. Dent. Assoc. 2016, 147, 255-263. [CrossRef] [PubMed]

152. Blotstein, F.A.; Jansen, E.C.; Jones, A.D.; Marshall, T.A.; Foxman, B. Dietary patterns associated with dental caries in adults in the United States. Community Dent. Oral Epidemiol. 2019, 48, 119-129. [CrossRef] [PubMed]

153. Antoniadou, M.; Varzakas, T. Diet and Oral Health Coaching Methods and Models for the Independent Elderly. Appl. Sci. 2020, 10, 4021. [CrossRef]

154. Antoniadou, M.; Varzakas, T. Breaking the vicious circle of diet, malnutrition and oral health for the independent elderly. Crit. Rev. Food Sci. Nutr. 2020, 1-23. [CrossRef]

155. Wärnberg-Gerdin, E.; Einarson, S.; Jonsson, M.; Aronsson, K.; Johansson, I. Impact of dry mouth conditions on oral health-related quality of life in older people. Gerodontology 2005, 22, 219-226. [CrossRef] [PubMed]

156. Schubert, M.M.; Izutsu, K.T. Iatrogenic Causes of Salivary Gland Dysfunction. J. Dent. Res. 1987, 66, 680-688. [CrossRef] [PubMed]

157. Scully, C. Drug effects on salivary glands: Dry mouth. Oral Dis. 2003, 9, 165-176. [CrossRef]

158. Ohara, Y.; Hirano, H.; Yoshida, H.; Obuchi, S.; Ihara, K.; Fujiwara, Y.; Mataki, S. Prevalence and factors associated with xerostomia and hyposalivation among community-dwelling older people in Japan. Gerodontology 2013, 33, 20-27. [CrossRef]

159. Saleh, J.; Zancanaro Figueiredo, M.A.; Cherubini, K.; Goncalves Salum, F. Salivary hypofunction: An update on aetiology, diagnosis and therapeutics. Arch. Oral Biol. 2015, 60, 242-255. [CrossRef] [PubMed]

160. Kudiyirickal, M.G.; Pappachan, J.M. Diabetes mellitus and oral health. Endocrine 2015, 49, 27-34. [CrossRef] [PubMed]

161. Poulopoulos, A.; Papadopoulos, P.; Andreadis, D. Chemotherapy: Oral side effects and dental interventions. A review of the literature. Stomatol. Dis. Sci. 2017, 1, 35-49. [CrossRef] 
162. Arrifin, A.; Heidari, E.; Burke, M.; Fenlon, M.R.; Banerjee, A. The Effect of Radiotherapy for Treatment of Head and Neck Cancer on Oral Flora and Saliva. Oral Health Prev. Dent. 2018, 16, 425-429. [CrossRef]

163. Kapila, Y.V.; Dodds, W.J.; Helm, J.F.; Hogan, W.J. Relationship between Swallow Rate and Salivary Flow. Dig. Dis. Sci. 1984, 29, 528-533. [CrossRef] [PubMed]

164. Tollosa, D.N.; Tavener, M.; Hure, A.; James, E.L. Adherence to multiple health behaviours in cancer survivors: A systematic review and meta-analysis. J. Cancer Surviv. 2019, 13, 327-343. [CrossRef]

165. Miller, W.D. Action of the Products of Fermentation on the Different Structures of the Mouth. In The Microorganisms of the Human Mouth. The Local and General Diseases Which Are Caused by Them; Miller, W.D., Ed.; Karger: Basel, Switzerland, 1973; pp. 119-145. ISBN 978-3-8055-1614-3.

166. Lingström, P.; Birkhed, D.; Ruben, J.; Arends, J. Effect of frequent consumption of starchy food items on enamel and dentin demineralization and on plaque $\mathrm{pH}$ in situ. J. Dent. Res. 1994, 73, 652-660. [CrossRef]

167. Lingström, P.; van Houte, J.; Kashket, S. Food starches and dental caries. Crit. Rev. Oral Biol. Med. 2000, 11, 366-380. [CrossRef]

168. Sheiham, A.; James, W.P.T. Diet and Dental Caries: The Pivotal Role of Free Sugars Reemphasized. J. Dent. Res. 2015, 94, 1341-1347. [CrossRef]

169. Toverud, G. The influence of war and post-war conditions on the teeth of Norwegian school children. II. Caries in the permanent teeth of children aged 7-8 and 12-13 years. Millbank Mem. Fund Q. 1957, 35, 127-196. [CrossRef]

170. Takeuchi, M. Epidemiological study on dental caries in Japanese children before, during and after World War II. Int. Dent. J. 1961, $11,443-457$.

171. Caglar, E.; Kuscu, O.O. The Role of Diet in Caries Prevention. In Evidence-Based Caries Prevention, 1st ed.; Eden, E., Ed.; Springer International Publishing: Cham, Switzerland, 2016; pp. 87-106.

172. Lingström, P.; Holm, A.K.; Mejare, I.; Twetman, S.; Söder, B.; Norlund, A.; Axelsson, S.; Lagerlöf, F.; Nordenram, G.; Petersson, L.G.; et al. Dietary factors in the prevention of dental caries: A systematic review. Acta Odontol. Scand. 2003, 61, 331-340. [CrossRef]

173. Moynihan, P.; Petersen, P.E. Diet, nutrition and the prevention of dental diseases. Public Health Nutr. 2004, 7, 201-226. [CrossRef] [PubMed]

174. Donohue, D.C.; Salminen, S. Safety of probiotic bacteria. Asia Pac. J. Clin. Nutr. 1996, 5, 25-28.

175. Berkey, D.; Berg, R. Geriatric oral health issues in the United States. Int. Dent. J. 2001, 51, 254-264. [CrossRef] [PubMed]

176. Bunker, J.P. The role of medical care in contributing to health improvements within societies. Int. J. Epidemiol. 2001, 30, 1260-1263. [CrossRef]

177. Mishra, S. Does modern medicine increase life-expectancy: Quest for the Moon Rabbit? Indian Heart J. 2016, 68, 19-27. [CrossRef]

178. Buxbaum, J.D.; Chernew, M.E.; Fendrick, A.M.; Cutler, D.M. Contributions of Public Health, Pharmaceuticals, and Other Medical Care to US Life Expectancy Changes, 1990-2015. Health Aff. 2020, 39, 1546-1556. [CrossRef]

179. Ezeh, A.C.; Bongaarts, J.; Mberu, B. Global population trends and policy options. Lancet 2012, 380, 142-148. [CrossRef]

180. Müller, F.; Naharro, M.; Carlsson, G.E. What are the prevalence and incidence of tooth loss in the adult and elderly population in Europe? Clin. Oral Implants Res. 2007, 18, 2-14. [CrossRef]

181. Slade, G.D.; Akinkugbe, A.A.; Sanders, A.E. Projections of U.S. Edentulism prevalence following 5 decades of decline. J. Dent. Res. 2014, 93, 959-965. [CrossRef]

182. Frencken, J. Caries Epidemiology and Its Challenges. In Caries Excavation: Evolution of Treating Cavitated Carious Lesions, Monographs in Oral Science; Schwendicke, F., Frencken, J., Innes, N., Eds.; Karger: Basel, Switzerland, 2018; Volume 27, pp. 11-23.

183. Thomson, W.M.; Ma, S. An ageing population poses dental challenges. Singap. Dent. J. 2014, 35, 3-8. [CrossRef] [PubMed]

184. Chen, S.; Wilson-Stanford, S.; Cromwell, W.; Hillman, J.D.; Guerrero, A.; Allen, C.A.; Sorg, J.A.; Smith, L. Site-Directed Mutations in the Lanthipeptide Mutacin 1140. Appl. Environ. Microbiol. 2013, 79, 4015-4023. [CrossRef]

185. Ghobrial, O.G.; Derendorf, H.; Hillman, J.D. Development and validation of a LC-MS quantification method for the lantibiotic MU1140 in rat plasma. J. Pharm. Biomed. Anal. 2009, 49, 970-975. [CrossRef] [PubMed]

186. Ghobrial, O.; Derendorf, H.; Hillman, J.D. Human serum binding and its effect on the pharmacodynamics of the lantibiotic MU1140. Eur. J. Pharm. Sci. 2010, 41, 658-664. [CrossRef]

187. Kirichenko, K.; Hillman, J.D.; Handfield, M.; Park, J.H. Complete synthesis of the bicyclic ring of a mutacin analog with orthogonally protected lanthionine via solid-phase intracyclization. J. Pept. Sci. 2019, 25, e3214. [CrossRef] [PubMed]

188. Declerck, D.; Leroy, R.; Martens, L.; Lesaffre, E.; Garcia-Zattera, M.J.; Vanden Broucke, S.; Debyser, M.; Hoppenbrouwers, K. Factors associated with prevalence and severity of caries experience in preschool children. Community Dent. Oral Epidemiol. 2008, 36, 168-178. [CrossRef] 